\title{
Acoustic Sources and Far-field Noise of Chevron and Round Jets
}

\author{
N.K. Depuru Mohan*, A.P. Dowling, S.A. Karabasov, H. Xia, O. Graham, \\ T.P. Hynes and P.G. Tucker \\ Department of Engineering, University of Cambridge, Cambridge CB2 1PZ, UK
}

This paper investigates numerically the acoustic sources and far-field noise of chevron and round jets. The acoustic sources are described by the fourth-order space-time velocity cross-correlations which are calculated based on a Large Eddy Simulation (LES) flowfield. Gaussian functions are found to fit the axial, radial and azimuthal cross-correlations reasonably well. The axial length scales are 3-4 times the radial and azimuthal length scales. For the chevron jet, the cross-correlation scales vary with azimuthal angle up to 6 jet diameters downstream; beyond that, they become axisymmetric like those for a round jet. The fourth-order space-time cross-correlation of the axial velocity, $\mathbf{R}_{1111}$, is the dominant source component and there are considerable contributions from other source components such as $\mathbf{R}_{2222}, \mathbf{R}_{3333}, \mathbf{R}_{1212}, \mathbf{R}_{1313}$ and $\mathbf{R}_{2323}$ cross-correlations where 1, 2 and 3 represent axial, radial and azimuthal directions respectively. For the chevron jet, these cross-correlations decay rapidly with axial distance whereas, for the round jet, they remain roughly constant over the first 10 jet diameters. The chevron jet intensifies both the $\mathbf{R}_{2222}$ and $\mathbf{R}_{3333}$ crosscorrelations within 2 jet diameters of the jet exit. The amplitude, length and time scales of the cross-correlations of a LES velocity field are investigated as functions of position and are found to be proportional to the turbulence amplitude, length and time scales that are determined from a Reynolds Averaged Navier-Stokes (RANS) calculation. The constants of proportionality are found to be independent of position within the jet and they are quite close for chevron and round jets. The scales derived from RANS are used for source description and an acoustic analogy is used for

*Corresponding author; AIAA Member; Email: nkd25@cam.ac.uk 
sound propagation. There is an excellent agreement between the far-field noise predictions and measurements. At low-frequencies, the chevron nozzle significantly reduces the far-field noise by $5-6 \mathrm{~dB}$ at $30^{0}$ and $2-3 \mathrm{~dB}$ at $90^{0}$ to the jet axis. However, the chevron nozzle slightly increases highfrequency noise. It was found that $\mathbf{R}_{1212}$ and $\mathbf{R}_{1313}$ cross-correlations have the largest contribution to the jet noise at $30^{0}$ to the jet axis whereas, the $\mathbf{R}_{2323}$ cross-correlation has the largest contribution to the jet noise at $\mathbf{9 0}^{0}$ to the jet axis. The RANS calculations are repeated with different turbulence models and the noise prediction is found to be almost insensitive to the turbulence model. The results indicate that the modelling approach is capable of assessing advanced noise-reduction concepts.

\section{Nomenclature}

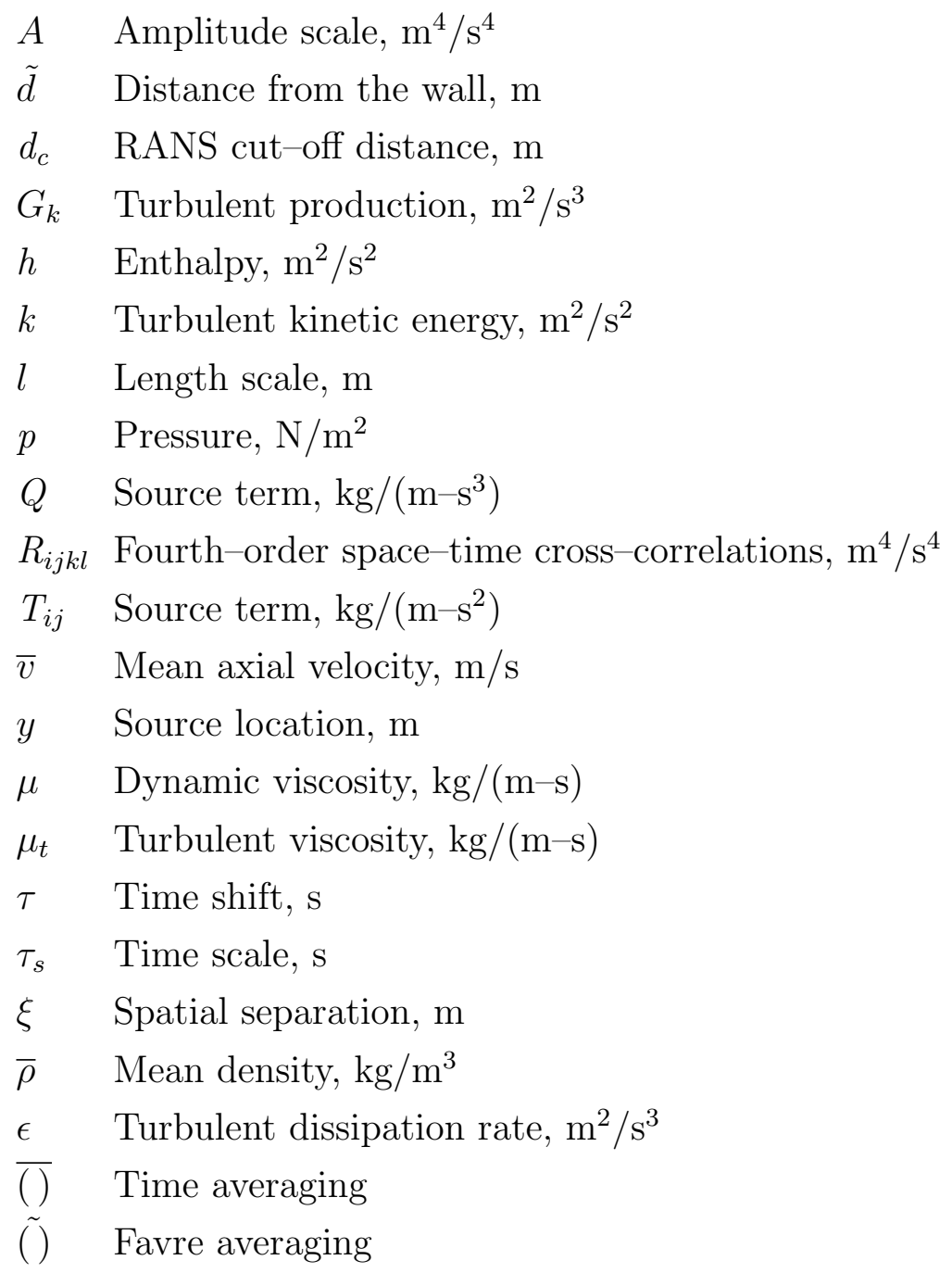




\section{Introduction}

Jet noise continues to be the dominant component of the overall aircraft noise at takeoff. To mitigate jet noise, there is a pressing need for advanced noise-suppression devices with a low thrust penalty. With the advent of chevron nozzles, many studies have been performed to maximise their benefit in terms of jet noise reduction by optimising their geometry. A parametric study of chevron nozzles was carried out by Bridges and Brown ${ }^{1}$ to explore the relationships among chevron geometric parameters, flow characteristics and far-field noise. Based on their experimental results, they concluded that chevron length has no major impact on either flow or noise; that chevron penetration significantly decreases noise at low frequencies and marginally increases noise at high frequencies; that chevron count should be kept at an optimum value of six for maximum benefit; and that chevron asymmetry reduces the impact of chevrons. They showed that hot jets differ systematically from cold jets but the overall trends with chevron parameters remain the same. They noticed that chevron penetration and chevron count are strong players with significant jet noise reduction at low frequencies. Gudmundsson and Colonius ${ }^{2}$ investigated the linear stability characteristics of chevron jets. They showed that the instability waves of chevron jets can be decomposed into azimuthal modes analogous to those of round jets. It was found that chevron jets have a higher number of unstable modes than their thrust-equivalent round jets. They noticed that the growth rate of chevron jets is significantly lower than round jets, which can be attributed to relatively thick shear layer of chevron jets. They observed that the low-frequency noise can be reduced by increasing chevron penetration.

Callendar et al. ${ }^{3}$ have shown that chevron count has low impact on noise radiation for high chevron count nozzles. Bridges and Brown ${ }^{1}$ also mentioned that there is an optimum count beyond which the impact of chevrons deteriorates in terms of jet noise reduction. Callendar et $a l .{ }^{4}$ showed that chevron nozzles have significantly reduced low-frequency noise for both high-shear and nominal-shear conditions. At high-frequencies, chevron nozzles have marginally increased far-field noise at high-shear conditions but surprisingly, they have slightly reduced far-field noise at nominal-shear conditions. They argued that though chevron nozzles increase high-frequency noise in the jet near-field, this does not necessarily mean there will be a comparable increase in the jet far-field because sound at high frequency is attenuated by propagation through the atmosphere. Fundamentally, any flow control device, such as chevrons, is intended to enhance mixing by increasing the turbulence intensity close to the nozzle. As chevrons generate strong streamwise vortices, it is expected to see high-frequency noise increase as it is a byproduct of vortex-enhanced mixing.

To investigate the effect of the chevron profile (curvature along the azimuthal direction) on the far-field noise, Tide and Srinivasan ${ }^{5}$ have performed experiments on various chevron 
nozzles for a wide range of Mach numbers. They compared the sinusoidal profile chevron nozzle with the conventional chevron nozzle which has a linear profile. In terms of the acoustic efficiency, the sinusoidal profile chevron nozzle has the highest impact on jet noise for supersonic flows. However, for subsonic flows, the sinusoidal profile chevron nozzle does not show any benefit compared to the conventional chevron nozzle.

Xia et $a l .{ }^{6}$ performed hybrid RANS-LES computations for chevron jets. The far-field noise was predicted by using the Ffowcs Williams-Hawkings acoustic analogy. To maintain numerical fidelity, they used a good quality mesh and did not introduce spurious sources. They showed very good agreement of mean and second-order fluctuating quantities of turbulence with measurements. Although there was a good agreement between the far-field noise predictions and measurements, there were some discrepancies at high frequencies. Engel et $a l^{7}$ demonstrated that RANS-based methods are reliable to obtain far-field noise predictions. RANS simulations were performed with a cubic $\mathrm{k}-\epsilon$ turbulence model and the far-field noise was predicted by Lighthill Ray Tracing method. The flow and noise predictions were in a reasonably good agreement with measurements. However, there were major discrepancies at low frequencies where chevrons are known to have impact on jet noise.

The Mani-Glibe-Balsa (MGB) method $^{8}$ predicts both aerodynamic and acoustic properties of turbulent jets. In this method, the jet aerodynamics were predicted using the Reichardt's turbulence model; mean flow-acoustic interaction effects were modelled using solutions to Lilley's equation; the relative contributions from the individual components of the source term were based on the quadrupole correlations. Although these showed a reasonably good agreement between predictions and measurements, there were some discrepancies at high-frequencies. Khavaran et al. ${ }^{9}$ generalised the aerodynamic component of MGB model by replacing Reichardt's turbulence model with $k-\epsilon$ turbulence model. This revised model has been named the MGB-Khavaran (MGBK) model. In addition, the effects of swirl, radial flow, shock structure and the flow path upstream of the nozzle exit were included. There was no change in the source description and modelling of mean flow-acoustic interaction effects. Predictions and measurements were in a reasonably good agreement though there were discrepancies at high-frequencies. Khavaran ${ }^{10}$ has reported two additional changes to the existing MGBK model. Firstly, an axisymmetric turbulence model was used rather than isotropic description of acoustic sources. Secondly, both types of source terms were included: shear-noise and self-noise. The effects of turbulence intensities on the radiated noise were also examined and the contributions of shear-noise and self-noise to the noise radiation were estimated. With these updates, they showed better noise predictions compared to MGBK model (1994). Viswanathan ${ }^{11}$ compared MGBK model (1999) and fine-scale turbulence mixing noise model proposed by Tam and Aurialt ${ }^{12}$ with his measurements. Tam and Auriault's model predicted the sound power spectral density better than the MGBK model. However, 
Tam and Aurialt's model does not work in the peak noise direction. They argued that the jet noise in the peak noise direction is due to large-scale turbulent structures rather than the fine-scale turbulence.

Freund et $a .^{13}$ evaluated the robustness of acoustic analogies: a uniform base flow (Lighthill-like) formulation, a parallel base flow (Lilley-like) formulation, a spreading mean flow formulation and a locally parallel approximation of this spreading mean flow formulation. They found that Lighthill-like formulation is considerably less robust to source errors than the others. Wang et al. ${ }^{14}$ reviewed computational techniques for predicting the flowgenerated noise. They focussed on hybrid approaches in which the turbulent noise sources are either computed or modelled separately from the far-field noise calculation. They showed that there are good reasons to expect that properly validated hybrid approaches will reduce the dependence on the experimental data and hence that they could advance the state of the art of computational aeroacoustics.

In the recent past, two approaches were proposed to overcome earlier problems with the classical acoustic analogies: Goldstein and Leib ${ }^{15}$ and Karabasov et al. ${ }^{16}$ The approach of Goldstein and Leib ${ }^{15}$ was based on Goldstein's ${ }^{17}$ generalised acoustic analogy. They obtained a system of linear equations by rearranging the equations of continuity, momentum, and energy and then split the problem into two parts: first find the vector Green's function for the system of equations, and model the statistical properties of acoustic sources. They emphasised that the slow divergence of the jet flow has to be accounted for in the Green's function. Symmetry properties and experimental data were used to describe the source statistical model. Karabasov et al. ${ }^{16}$ also used Goldstein's ${ }^{17}$ generalised acoustic analogy, but they calculated the vector Green's function numerically using adjoint approach proposed by Tam and Aurialt. ${ }^{18}$ They described the acoustic sources by calculating fourth-order space-time cross-correlations based on a LES flowfield. They used Gaussian functions to model the source cross-correlations and included important effects such as the divergence of the mean flow, the anisotropy of the source and the radial variation of the Green's function. Although these two approaches are based on different assumptions, they both achieved good agreement with experiments.

This study investigates numerically the acoustic sources and far-field noise of chevron and round jets. The modelling approach is based on Karabasov et al. ${ }^{16}$ and includes both flow and acoustic modelling. Further details are given in $\S$ II and III. The SMC006 chevron nozzle and the SMC000 round nozzle (figure 1) are chosen from a parametric study conducted by Bridges and Brown. ${ }^{1}$ This particular chevron nozzle is chosen as it was identified as the most effective for jet noise reduction. Both nozzles are thrust-equivalent and their exit flow conditions are maintained at: Reynolds number $=1.03 \times 10^{6}$; jet Mach number $=0.9$ and temperature ratio (the ratio of the jet static temperature and the ambient temperature) $=$ 
0.84. The geometric details of the considered chevron and round nozzles are provided in table 1. The effective diameter was determined experimentally by assuming the discharge coefficient to be the same for both nozzles and the measured mass flow was used as a surrogate area measurement.

\begin{tabular}{|c|c|c|c|c|c|}
\hline $\begin{array}{c}\text { Nozzle } \\
\text { ID }\end{array}$ & $\begin{array}{c}\text { Chevron } \\
\text { Count } \\
\text { N }\end{array}$ & $\begin{array}{c}\text { Chevron } \\
\text { Length } \\
\mathrm{L} \\
(\mathrm{mm})\end{array}$ & $\begin{array}{c}\text { Chevron } \\
\text { Angle } \\
\theta \\
\text { (deg) }\end{array}$ & $\begin{array}{c}\text { Chevron } \\
\text { Penetration } \\
\mathrm{P} \\
(\mathrm{mm})\end{array}$ & $\begin{array}{c}\text { Effective } \\
\text { Diameter } \\
\mathrm{D}_{\text {eff }} \\
(\mathrm{mm})\end{array}$ \\
\hline SMC000 (Round) & 0 & - & - & - & 50.8 \\
\hline SMC006 (Chevron) & 6 & 22.6 & 18.2 & 3.525 & 47.7 \\
\hline
\end{tabular}

Table 1: Geometric details of the considered nozzles

\section{Flow Modelling}

\section{II.A. LES Modelling}

A hybrid LES-RANS modelling (Xia et $a l .{ }^{19}$ ) is used to simulate both the chevron and round jet flows. The Favre-average Navier-Stokes equations are solved using a parallel finite volume in-house code. The Spalart-Allamaras RANS model is used near the nozzle wall. A level set type approach is used to calculate the wall distance. Based on this wall distance, the LES and RANS zones are defined and they are blended by the Hamilton-Jacobi equation:

$$
|\nabla \tilde{d}|=1+f(\tilde{d}) \nabla^{2} \tilde{d}+g(\tilde{d})
$$

where $f(\tilde{d})=\epsilon_{0} \tilde{d} ; g(\tilde{d})=\epsilon_{1}\left(\tilde{d} / d_{c}\right)^{2} ; \tilde{d}$ is the distance from the wall and $d_{c}$ is the RANS cut-off distance. As the flow equations are solved in parallel, this equation is solved as an additional equation to reduce the computational cost of the search for the nearest wall distance.

The LES cases were run for approximately 300,000 time steps, with a physical time step of $1.7 \times 10^{-7}$ seconds (maximum CFL number is around 0.3 ). It took around 100,000 time steps to reach a fully-developed jet, another 100,000 time steps to capture the turbulence scales, and a further 100,000 time steps to obtain the cross-correlations of the turbulent velocity field that describe the acoustic source. The extent of the three-dimensional LES computational domain is 72 jet diameters in the axial direction and 50 jet diameters in the radial direction for the entire $360^{\circ}$ of the jet. The grid for a single chevron is generated first and replicated azimuthally. The assessment of mesh sensitivity is done by comparing the flow results of 12 and 20 million-node meshes and the latter showed slightly better agreement with 
experiments. Therefore, the 20 million-node mesh with appropriate boundary conditions (Xia and Tucker ${ }^{6}$ ) is chosen. Multi-block type hexahedral grids were used to achieve high mesh accuracy. To avoid clustering of polar points or lines, a singularity treatment is done along the jet centreline. The best practices ${ }^{20}$ were followed to maintain numerical fidelity.

\section{II.B. RANS Modelling}

The commercial software, ANSYS13.0 FLUENT, is used to perform RANS steady flow calculations for both the chevron and round jets. As the jet flow is highly turbulent i.e. Reynolds number $=1.03 \times 10^{6}$, the standard $k-\epsilon$ turbulence model is chosen first for the jet flow calculations. The extent of the RANS computational domain is the same as that of the LES, but only a $60^{\circ}$ sector is considered as the jet mean flow is identical for each chevron. The mesh sensitivity is evaluated by $0.5,2.5$ and 5 million-node meshes and there was no considerable difference in the jet flow results. Therefore, the 0.5 million-node mesh is chosen as it provides quicker and reasonably accurate results.

The FLUENT software obtains the jet flowfield by solving the compressible Navier-Stokes equation given below,

$$
\frac{\partial}{\partial t}\left(\rho v_{i}\right)+\frac{\partial}{\partial x_{j}}\left(\rho v_{i} v_{j}\right)=\frac{\partial \sigma_{i j}}{\partial x_{j}}
$$

where

$$
\sigma_{i j}=-p \delta_{i j}+\mu\left(\frac{\partial v_{i}}{\partial x_{j}}+\frac{\partial v_{j}}{\partial x_{i}}\right)
$$

where $p$ is pressure, $v_{i}$ is velocity, $\mu$ is dynamic viscosity and $\rho$ is density. Although this study is limited to unheated jets, the energy equation is solved.

$$
\frac{\partial E}{\partial t}+\frac{\partial\left((E+p) v_{i}\right)}{\partial x_{i}}=0
$$

where $E=\rho e+0.5 \rho\left(u^{2}+v^{2}+w^{2}\right)$ is the total energy per unit volume, with $e$ being the internal energy per unit mass of the fluid.

The standard $k-\epsilon$ turbulence model is based on the transport equations of turbulent kinetic energy, $k$ and its rate of dissipation, $\epsilon$. The transport equation for turbulent kinetic energy, $k$, is derived from the exact equation, whereas the transport equation for rate of dissipation of turbulent kinetic energy, $\epsilon$, is obtained using physical reasoning. The transport equations for turbulent kinetic energy, $k$, and turbulent dissipation, $\epsilon$, are:

$$
\frac{\partial}{\partial t}(\rho k)+\frac{\partial}{\partial x_{i}}\left(\rho k v_{i}\right)=\frac{\partial}{\partial x_{j}}\left[\left(\mu+\frac{\mu_{t}}{\sigma_{k}}\right) \frac{\partial k}{\partial x_{j}}\right]+G_{k}-\rho \epsilon
$$


and

$$
\frac{\partial}{\partial t}(\rho \epsilon)+\frac{\partial}{\partial x_{i}}\left(\rho \epsilon v_{i}\right)=\frac{\partial}{\partial x_{j}}\left[\left(\mu+\frac{\mu_{t}}{\sigma_{\epsilon}}\right) \frac{\partial \epsilon}{\partial x_{j}}\right]+C_{1 \epsilon} \frac{\epsilon}{k} G_{k}-C_{2 \epsilon} \rho \frac{\epsilon^{2}}{k}
$$

where $v_{i}$ is mean velocity; $G_{k}$ is turbulent production; $\mu$ is dynamic viscosity; $\mu_{t}$ is turbulent viscosity which is an added viscosity that appears due to the way turbulence is represented. It is defined as: $\mu_{t}=\rho C_{\mu} k^{2} / \epsilon$ where $C_{\mu}=0.09$. In our RANS calculations, $C_{1 \epsilon}$ $=1.44 ; C_{2 \epsilon}=1.92 ; \sigma_{k}=1$ and $\sigma_{\epsilon}=1.3$ are taken as the turbulent Prandtl numbers for $k$ and $\epsilon$ respectively.

To examine the effect of turbulence models on far-field noise predictions, various turbulence models such as RNG and realisable $k-\epsilon$, standard and SST $k-\omega$, Reynolds Stress models are considered. The RANS scales based on $k-\epsilon$ turbulence models are defined as: amplitude scale $=(2 \bar{\rho} k)^{2} ;$ time scale $=k / \epsilon$ and length scale $=k^{3 / 2} / \epsilon$. The RANS scales based on $k-\omega$ turbulence models are defined as: amplitude scale $=(2 \bar{\rho} k)^{2}$; time scale $=1 /(0.09 \omega)$ and length scale $=k^{1 / 2} / \omega$ where $k$ is turbulent kinetic energy; $\epsilon$ is turbulent dissipation rate and $\omega$ is turbulent eddy frequency $=0.09 \epsilon / k$ (Launder and Spalding ${ }^{21}$ ). The effects of different turbulence models on the correlation scales and far-field noise are investigated in $\S$ IV.C.7.

\section{Acoustic Modelling}

\section{III.A. Acoustic Sources}

In this study, the following steps are followed to describe the acoustic sources:

1. The fourth-order space-time velocity cross-correlations are described as

$$
R_{i j k l}(\mathbf{y}, \xi, \tau)=\overline{T_{i j}^{\prime}(\mathbf{y}, t) T_{k l}^{\prime}(\mathbf{y}+\xi, t+\tau)}
$$

where

$$
T_{i j}^{\prime}=-\left(\rho v_{i}^{\prime \prime} v_{j}^{\prime \prime}-\bar{\rho} \widetilde{v_{i}^{\prime \prime} v_{j}^{\prime \prime}}\right)
$$

where $\mathbf{y}$ is the source location; $v^{\prime \prime}$ is fluctuating velocity; $\tau$ is time shift; $\xi$ is spatial separation; $\rho$ is fluid density, $t$ is time and $\widetilde{(~)}$ represents Favre averaging.

2. The shape of these cross-correlations are approximated as the Gaussian function:

$$
\begin{aligned}
\mathrm{R}_{i j k l}(\mathbf{y}, \xi, \tau)=\mathrm{A}_{i j k l, L E S}(\mathbf{y}) \exp \left[-\frac{\xi_{1}}{\bar{v}(\mathbf{y}) \tau_{L E S}(\mathbf{y})}\right] \\
\exp \left[-\ln 2\left(\frac{\left(\xi_{1}-\bar{v}(\mathbf{y}) \tau\right)^{2}}{l_{1, L E S}(\mathbf{y})^{2}}+\frac{\xi_{2}^{2}}{l_{2, L E S}(\mathbf{y})^{2}}+\frac{\xi_{3}^{2}}{l_{3, L E S}(\mathbf{y})^{2}}\right)\right]
\end{aligned}
$$

where $\mathrm{A}_{i j k l, L E S}$ is an amplitude scale; $\tau_{L E S}$ is a time scale; $l_{1, L E S}, l_{2, L E S}$ and $l_{3, L E S}$ 
are axial, radial and azimuthal length scales respectively; $\xi_{1}, \xi_{2}$ and $\xi_{3}$ are spatial separations in the axial, radial and azimuthal directions respectively; $\bar{v}$ is the mean axial velocity at location $\mathbf{y}$ and $\tau$ is time shift. The parameters $\mathrm{A}_{i j k l, L E S}, \tau_{L E S}, l_{1, L E S}, l_{2, L E S}$ and $l_{3, L E S}$ are determined by curve fitting (using a non-linear least squares method) the Gaussian form onto the cross-correlations that are calculated based on a LES flowfield.

3. The relative magnitudes of $\mathrm{R}_{i j k l}$ cross-correlations with respect to the dominant crosscorrelation and their variation with nozzle geometry and source position within the jet is investigated in $\S$ IV.B.4.

4. RANS scales are first calculated based on the standard $k-\epsilon$ turbulence model: amplitude scale, $\mathrm{A}_{R A N S}=(2 \bar{\rho} k)^{2}$; time scale, $\tau_{R A N S}=k / \epsilon$ and length scale, $l_{R A N S}=k^{3 / 2} / \epsilon$ where $\bar{\rho}$ is mean density; $k$ is turbulent kinetic energy and $\epsilon$ is turbulent dissipation rate.

5. The constants of proportionality between LES and RANS scales are defined as: $\mathrm{A}_{i j k l, L E S}$ $=\mathrm{C}_{i j k l} * A_{R A N S} ; l_{i, L E S}=\mathrm{C}_{l i} * l_{R A N S}$ and $\tau_{L E S}=\mathrm{C}_{\tau} * \tau_{R A N S}$ and are taken to be constant throughout the jet.

6. The RANS scales multiplied by the determined proportionality constants are used for the source description.

By following these steps, RANS scales can be used to accurately describe the source cross-correlations. This source description is then used in the sound propagation model to predict the far-field noise.

\section{III.B. Sound Propagation}

In this study, the far-field noise is predicted using Karabasov et al. ${ }^{16}$ approach which can be briefly summarised as:

1. The instantaneous form of flow properties is decomposed into mean and fluctuating quantities (Goldstein ${ }^{17}$ decomposition).

$$
\rho=\bar{\rho}+\rho^{\prime} \quad p=\bar{p}+p^{\prime} \quad v_{i}=\tilde{v}_{i}+v_{i}^{\prime \prime} \quad h=\tilde{h}+h^{\prime \prime}
$$

where $\overline{()}$ represents time averaging, () represents Favre averaging, single and double primes represent the corresponding fluctuations, $\rho$ is density, $p$ is pressure, $v_{i}$ is velocity and $h$ is enthalpy. 
2. The Navier-Stokes equations can then be re-arranged into Linearised Euler Equations (LEEs) where the linear terms are kept on the left-hand side representing noise propagation, and the non-linear terms on the right-hand side representing noise sources.

$$
\begin{gathered}
\frac{\partial \rho^{\prime}}{\partial \tau}+\frac{\partial}{\partial y_{j}}\left(\rho^{\prime} \tilde{v}_{j}+u_{j}\right)=0 \\
\frac{\partial u_{i}}{\partial \tau}+\frac{\partial}{\partial y_{j}}\left(\tilde{v_{j}} u_{i}\right)+\frac{\partial p^{\prime}}{\partial y_{i}}+u_{j} \frac{\partial \tilde{v}_{i}}{\partial y_{j}}-\left(\frac{\rho^{\prime}}{\bar{\rho}}\right) \frac{\partial \tilde{\tau_{i j}}}{\partial y_{j}}=\frac{\partial T_{i j}^{\prime}}{\partial y_{j}} \\
\left(\frac{1}{\gamma-1}\right) \frac{\partial p^{\prime}}{\partial \tau}+\left(\frac{1}{\gamma-1}\right) \frac{\partial}{\partial y_{j}}\left(p^{\prime} \tilde{v_{j}}\right)+\frac{\partial}{\partial y_{j}}\left(u_{j} \tilde{h}\right)+p^{\prime} \frac{\partial \tilde{v}_{j}}{\partial y_{j}}-\left(\frac{u_{i}}{\bar{\rho}}\right) \frac{\partial \tilde{\tau_{i j}}}{\partial y_{j}}=Q
\end{gathered}
$$

Definitions:

Momentum perturbation variable, $u_{i}=\rho v_{i}^{\prime \prime}$

Favre-averaged stagnation enthalpy, $\tilde{h}_{0}=\tilde{h}+0.5 \times \tilde{v}^{2}$

Perturbation of stagnation enthalpy, $h_{0}^{\prime \prime}=h^{\prime \prime}+\tilde{v}_{i} v_{i}^{\prime \prime}+0.5 \times v^{\prime \prime 2}$

3. The source terms are given by,

$$
\begin{gathered}
T_{i j}^{\prime}=-\left(\rho v_{i}^{\prime \prime} v_{j}^{\prime \prime}-\bar{\rho} \widetilde{v_{i}^{\prime \prime} v_{j}^{\prime \prime}}\right) \\
Q=-\tilde{v}_{j} \frac{\partial T_{i j}^{\prime}}{\partial y_{i}}+\frac{1}{2} \delta_{i j}\left[\frac{D T_{i j}^{\prime}}{D \tau}+\frac{\partial \tilde{v_{k}}}{\partial y_{k}} T_{i j}^{\prime}\right]-\frac{\partial}{\partial y_{j}}\left(\rho v_{j}^{\prime \prime} h_{0}^{\prime \prime}-\bar{\rho} v_{j}^{\prime \prime} h_{0}^{\prime \prime}\right)
\end{gathered}
$$

where $D / D \tau$ is the convective derivative, which is given by

$$
\frac{D}{D \tau}=\frac{\partial}{\partial \tau}+\tilde{v}_{j}(\mathbf{y}) \frac{\partial}{\partial y_{j}} .
$$

As this study is limited to unheated jets, the last term of equation 15 is negligible and the source term, $Q$, can be simplified to

$$
Q=-\tilde{v}_{j} \frac{\partial T_{i j}^{\prime}}{\partial y_{i}}+\frac{1}{2} \delta_{i j}\left[\frac{D T_{i j}^{\prime}}{D \tau}+\frac{\partial \tilde{v}_{k}}{\partial y_{k}} T_{i j}^{\prime}\right]
$$

4. The wave propagation problem is solved in the frequency domain using an adjoint Green's function method (Tam and Aurialt ${ }^{18}$ ). 
5. The Fourier transform of adjoint Green's function is expressed as the sum of a freespace solution for a point source and a scattered field due to the jet flow. The free-space solution of a point source is known analytically, and the scattered field is found in the frequency domain.

6. The source term on the right-hand side of Linearised Euler Equations not only generates sound but may also produce exponentially growing instability waves that can grow on the jet shear layers. But, these have already been included in the source term. Hence, the solution required for the Green's function should not contain any part of the shear-layer instability solution (Agarwal et al. ${ }^{22}$ ). In the time domain, this is equivalent to requiring that the Green's function be weakly casual and remain finite in the far-field (Dowling et $a .^{23}$ ). To ensure this, a dual-scale iterative pseudo-time stepping scheme (Karabasov and Hynes ${ }^{24}$ ) is used.

7. The representation theorem is then formulated for the far-field sound pressure in terms of the Green's function and source terms.

$$
\hat{p}(\mathbf{x}, \omega)=-\int_{V_{\infty}(\mathbf{y})}\left(\hat{G}_{i}(\mathbf{y},-\omega \mid \mathbf{x}) \frac{\partial \hat{T}_{i j}^{\prime}}{\partial y_{j}}(\mathbf{y}, \omega)+\hat{G}_{4}(\mathbf{y},-\omega \mid \mathbf{x}) \hat{Q}(\mathbf{y}, \omega)\right) d^{3} \mathbf{y}
$$

where $\left(\hat{)}\right.$ represents the Fourier transform; $G_{0}$ is an adjoint density-like variable; $G_{1-3}$ are adjoint momentum-like variables; $G_{4}$ is an adjoint pressure-like variable; $T_{i j}^{\prime}$ and $Q$ are source terms. After expanding $\hat{Q}(\mathbf{y}, \omega)$ as in the equation 17 and then performing integration by parts, we obtain

$$
\hat{p}(\mathbf{x}, \omega)=-\int_{V_{\infty}(\mathbf{y})} \hat{I}_{i j}(\mathbf{y},-\omega \mid \mathbf{x}) \hat{T}_{i j}^{\prime}(\mathbf{y}, \omega) d^{3} \mathbf{y}
$$

where $\hat{I}_{i j}$, the Fourier transform of a second-rank wave propagation tensor, is given by,

$$
\begin{gathered}
\hat{I}_{i j}(\mathbf{y}, \omega \mid \mathbf{x})=\frac{\partial \hat{G}_{j}}{\partial y_{i}}(\mathbf{y}, \omega \mid \mathbf{x})-\left[\frac{\partial \tilde{v}_{j}}{\partial y_{i}}(\mathbf{y}) \hat{G}_{4}(\mathbf{y}, \omega \mid \mathbf{x})+\tilde{v}_{j}(\mathbf{y}) \frac{\partial \hat{G}_{4}}{\partial y_{i}}(\mathbf{y}, \omega \mid \mathbf{x})\right] \\
+\frac{\delta_{i j}}{2}\left(i \omega+\tilde{v}_{k} \frac{\partial}{\partial y_{k}}\right) \hat{G}_{4}(\mathbf{y}, \omega \mid \mathbf{x})
\end{gathered}
$$

8. The sound power spectral density can be expressed as,

$$
\hat{P}(\mathbf{x}, \omega)=\int_{V_{\infty}(\mathbf{y})} \int_{\xi} \hat{R}_{i j k l}(\mathbf{y}, \xi, \omega) \hat{I}_{i j}(\mathbf{y}, \omega \mid \mathbf{x}) \hat{I}_{k l}(\mathbf{y}+\xi,-\omega \mid \mathbf{x}) d^{3} \xi d^{3} \mathbf{y}
$$


where $\hat{I}_{i j}$ and $\hat{I}_{k l}$ are the Fourier transform of second-rank wave propagation tensors and $\hat{R}_{i j k l}$ is the Fourier transform of fourth-order space-time cross-correlations, which is given by

$$
\hat{R}_{i j k l}(\mathbf{y}, \xi, \omega)=\int R_{i j k l}(\mathbf{y}, \xi, \tau) e^{-i \omega \tau} d \tau=\int \overline{T_{i j}^{\prime}(\mathbf{y}, t) T_{k l}^{\prime}(\mathbf{y}+\xi, t+\tau)} e^{-i \omega \tau} d \tau
$$

In this study, the local mean flow is a function of radial and axial position, but an average over the azimuthal direction is used for the sound propagation calculations. The sound propagation from the acoustic sources in the jet near-field to the far-field is determined through the calculation of a Green's function. The Green's function is the solution of the adjoint Linearised Euler Equations (LEEs). With a numerical solution, the effects of scattering by the nozzle and the axial development of the jet mean flow can be included. Instead for simplicity, a locally parallel jet flow approximation is made in this study so that the Green's function can be quickly calculated analytically.

\section{Results and Discussion}

\section{IV.A. Aerodynamics}

\section{IV.A.1. Centreline Velocity}

The decay rate of centreline velocity provides an indication of how effectively a chevron nozzle mixes the jet with the ambient air i.e. the faster the centreline velocity decays, the better the turbulent mixing. Figure 2 shows that the centreline velocity decays dramatically beyond 4 jet diameters for the chevron jet, whereas the round jet centreline velocity only starts decaying after 6 jet diameters. The chevron jet reduces the potential core length by approximately $33 \%$ compared to that of the round jet. This demonstrates the strong influence of a chevron nozzle over the jet flowfield. This drastic reduction in the potential core length enhances turbulent mixing within 6 jet diameters from the nozzle exit. The LES predictions show excellent agreement with Bridges and Brown ${ }^{1}$ measurements, whereas the RANS predictions are reasonably good.

\section{IV.A.2. Turbulence Intensity}

The turbulence intensity is defined as the ratio of the fluctuating axial velocity to the mean axial velocity,

$$
I=\frac{u_{r m s}^{\prime}}{\bar{u}}
$$


where $u_{r m s}^{\prime}$ is the root mean square of fluctuating axial velocity and $\bar{u}$ is the mean axial velocity.

The turbulence intensity on the jet centreline for round and chevron jets are compared in figure 3. In both cases, the maximum turbulence intensity occurs 1-2 jet diameters after the axial location at which the jet centreline velocity starts to decay (see figure 2). The peak turbulence intensity could therefore be due to the break-down of the jet potential core. As the chevron jet has a shorter potential core, the axial location of its peak turbulence intensity is 3-4 jet diameters before to that of the round jet. This indicates that chevrons increase the turbulence intensity in the jet near-field.

\section{IV.A.3. Streamwise Vorticity}

The streamwise vorticity is defined as,

$$
\omega_{x}=\left(\frac{\partial \bar{v}}{\partial z}-\frac{\partial \bar{w}}{\partial y}\right)
$$

where $\bar{v}$ is the mean radial velocity and $\bar{w}$ is the mean azimuthal velocity.

The generation of streamwise vortices is one of the most effective mechanisms to enhance turbulent mixing. Figure 4 shows that the chevron jet generates strong streamwise vortices, whereas the round jet does not. As the jet flow is obstructed by the chevron tips, it tries to escape around the chevron slants and through the chevron roots. This generates two counter-rotating streamwise vortices of equal strength at each chevron, anti-symmetrical about the chevron tips. These streamwise vortices enhance the mixing between the jet and ambient air and then dissipate by $4-5$ jet diameters downstream of the nozzle exit. The chevron nozzle works as a flow control device through the generation of streamwise vortices. This vortex-enhanced mixing lowers the relative velocity between the jet and ambient air. According to Lighthill's ${ }^{25,26}$ eighth power law, this reduces the far-field jet noise levels.

\section{IV.A.4. Spreading Rate}

The spreading rate is defined as,

$$
S=\frac{d r_{1 / 2}}{d x}
$$

where $r_{1 / 2}$ is the jet half radius i.e. a radial distance where the mean axial velocity falls to half of centreline velocity.

The spreading rate of the chevron jet is a function of azimuthal angle for the first 6 jet diameters from the nozzle exit. Figure 5 shows that the spreading rate downstream of the chevron roots is greater than that downstream of the chevron tips. The spreading rate half way between these two angles, a location we refer to as the chevron slants, is very similar 
to that of the round jet. It should be noted that spreading rate downstream of the chevron roots varies non-linearly with axial distance. This can be explained as the spreading rate downstream of the roots increases until it is influenced by the effects of flow past adjacent chevron tips. Thereafter, the spreading rate downstream of the chevron roots decreases until 4 jet diameters and then slowly transitions to a linear behaviour. Beyond 6 jet diameters from the nozzle exit, both the chevron and round jets spread linearly with axial distance at a spreading rate of 0.11 (figure 6). The non-linear spreading of the chevron jet with axial distance is better captured by LES compared to RANS. However, RANS is capable of capturing the linear behaviour of the jet spreading rate with axial distance.

In general, the LES predictions matched the experiments conducted by Bridges and Brown $^{1}$ within $5 \%$. The RANS predictions showed a reasonably good agreement with the experiments. The RANS flowfield will be used as an input for the acoustic calculations except that the acoustic source is LES-informed i.e. the relative magnitudes of $\mathrm{R}_{i j k l}$ crosscorrelations with respect to the $\mathrm{R}_{1111}$ cross-correlation are taken from the LES flowfield.

\section{IV.B. Noise Sources}

\section{IV.B.1. Cross-correlations}

Figures 7-12 show that Gaussian functions fit the $\mathrm{R}_{1111}$, the fourth-order space-time crosscorrelations of axial velocity, reasonably well for both the chevron and round jets. The shift in the peak of the cross-correlation for axial separations is due to strong convection in that direction. The cross-correlation decays rapidly with radial distance. As the presence of chevrons introduce angular variations in the jet flow, there is a considerable difference in cross-correlations with azimuthal angle. Cross-correlations for radial and azimuthal separations do not experience the peak shift as there is no strong convection in those directions.

The magnitude of the $\mathrm{R}_{1111}$ cross-correlation is higher for the round jet compared to the chevron jet. In addition, the decay rate of cross-correlation with respect to axial distance is slower for the round jet than for the chevron jet. This implies that the dominant source component, the $\mathrm{R}_{1111}$ cross-correlation, is stronger in the round jet than for the chevron jet.

\section{IV.B.2. Length Scales}

The length scales in the Gaussian description of the $\mathrm{R}_{1111}$ cross-correlation are compared for the chevron and round jets in tables 2 and 3 respectively. The axial correlation length is the largest length scale for both the chevron and round jets. For the chevron jet, the axial length scale is three times the radial and azimuthal length scales. The relative magnitudes of the radial or azimuthal length scales depend on axial location. The azimuthal length scale is larger than the radial length scale within 2 jet diameters from the nozzle exit whereas, 
beyond 8 jet diameters this is reversed. It is probable that the strong streamwise vortices generated in the chevron jet affect these length scales. As streamwise vortices decay rapidly along the axial direction, the radial length scale becomes larger than the azimuthal length scale beyond 8 jet diameters downstream.

For the round jet, the axial length scale is 3-4 times the radial or azimuthal length scales. The round jet does not have significant streamwise vorticity. It is possible that the entrainment of the ambient air into the jet leads to larger length scales in the radial direction compared to that in the azimuthal direction, particularly close to the nozzle.

\begin{tabular}{ccc}
\hline $\mathrm{x} / \mathrm{D}$ & Radial/Axial & Azimuthal/Axial \\
\hline 2 & 0.29 & 0.39 \\
4 & 0.34 & 0.36 \\
8 & 0.36 & 0.29 \\
10 & 0.34 & 0.30 \\
\hline
\end{tabular}

Table 2: SMC006 - Comparison of length scales

\begin{tabular}{ccc}
\hline $\mathrm{x} / \mathrm{D}$ & Radial/Axial & Azimuthal/Axial \\
\hline 2 & 0.33 & 0.24 \\
4 & 0.31 & 0.22 \\
8 & 0.28 & 0.26 \\
10 & 0.25 & 0.25 \\
\hline
\end{tabular}

Table 3: SMC000 - Comparison of length scales

\section{IV.B.3. Proportionality Constants}

One of our objectives is to use a RANS flowfield instead of an LES flowfield for fast and accurate far-field jet noise predictions. To achieve this, we test whether the RANS length, time and amplitude scales capture the variation of the cross-correlation scales determined from the LES. The amplitude scale drastically decays with axial distance for the chevron jet, whereas it remains roughly constant for the round jet. The length and time scales increase linearly with axial distance for both the chevron and round jets.

There is a relationship among scales at azimuthal angles in line with the chevron tips, roots and slants at a fixed axial location. Examining the length scales, it is observed that they are largest at chevron tips and smallest at chevron roots i.e. $l_{\text {tip }}>l_{\text {slant }}>l_{\text {root }}$. The same trend is observed in the time scale as well. However, the amplitude scales have a different trend; chevron slants have the highest amplitudes followed by chevron tips and then chevron roots. These relationships hold up to 6 jet diameters from the nozzle exit, and beyond that these scales become equal i.e. they become axisymmetric like a round jet. 
Figures 13-18 show that the RANS scales multiplied by fixed proportionality constants are a good fit to the LES-determined Gaussian parameters. The proportionality constant for the amplitude scale, $\sqrt{\mathrm{C}_{1111}}=\sqrt{\mathrm{R}_{1111}(\mathrm{x}, 0,0)} /(2 \bar{\rho} k)$, is 0.25 for both the chevron and round jets. The proportionality constants for axial, radial and azimuthal length scales, $\mathrm{C}_{l i}=$ $l_{i} /\left(k^{1.5} / \epsilon\right)$, is $0.30,0.09,0.09$ and $0.22,0.07,0.07$ for the chevron and round jets respectively. The proportionality constant for time scale, $\mathrm{C}_{\tau}=\tau /(k / \epsilon)$, is 0.16 and 0.12 for the chevron and round jets respectively. The RANS scales that are scaled by these proportionality constants are comparable to the LES scales throughout the jet and hence can be used for the accurate description of the source cross-correlation function. The proportionality constants of the chevron and round jets are quite close in spite of significant differences between their nozzle geometries; this clearly indicates that they are weakly dependent on nozzle geometry. The proportionality constant for the amplitude scale, $\sqrt{\mathrm{C}_{1111}}$, is 0.25 for both the chevron and round jets. The average values of the proportionality constants are used for both chevron and round jets, $\mathrm{C}_{l 1}=0.26, \mathrm{C}_{l 2}=0.08, \mathrm{C}_{l 3}=0.08$ and $\mathrm{C}_{\tau}=0.14$, for their source description.

\section{IV.B.4. Major Cross-correlations}

Along the lip line $(\mathrm{r}=0.5 \mathrm{D})$, the auto-correlation functions, $\mathrm{R}_{i j k l}(\mathbf{x}, \mathbf{0}, 0)$, reduce dramatically with axial distance for the chevron jet whereas, they appear to be roughly constant up to 10 jet diameters downstream from the nozzle exit for the round jet (figure 19). This indicates that the major noise sources of the chevron jet are concentrated close to nozzle while they have a greater axial extent for the round jet. The magnitude of the auto-correlation functions for the chevron jet after the rapid decay is much lower compared to that of the round jet. This confirms that the acoustic source itself is much stronger for the round jet compared to the chevron jet. In other words, chevrons affect the jet flowfield in such a way that they drastically weaken the dominant (largest) acoustic source, the $\mathrm{R}_{1111}$ crosscorrelation, by 50-60\% compared to that of the round jet. This clearly demonstrates the impact of chevron nozzles in terms of jet noise reduction, which indeed have brought a significant change in the aircraft noise reduction technology.

As $R_{1111}$ is the dominant component of the source cross-correlation function, the other source cross-correlations are normalised by $\mathrm{R}_{1111}$ to identify the major noise sources (figure 20). $R_{2222}$ and $R_{3333}$ are the next largest components of the source cross-correlation function. The other major source cross-correlations are: $R_{1212}, R_{1313}$ and $R_{2323}$ and other terms they are equal to by symmetry. The major components of the source cross-correlation function remain the same for both the chevron and round jets. The chevron nozzle intensifies both $\mathrm{R}_{2222}$ and $\mathrm{R}_{3333}$ close to nozzle. These relative magnitudes of $\mathrm{R}_{i j k l}(\mathbf{x}, \mathbf{0}, 0)$ with respect to $\mathrm{R}_{1111}(\mathbf{x}, \mathbf{0}, 0)$ are calculated from the LES flowfield and they are used in the source description. This means that the source modelling is informed by the LES. 
A comparison study was performed to investigate the auto-correlation of major sources (tables 4 and 5 ). In all cases, $R_{2222}$ and $R_{3333}$ are approximately equal. In case of the chevron jet, $R_{1111}$ is approximately twice $R_{2222}$ or $R_{3333}$ within 2 jet diameters downstream and three times it beyond 8 jet diameters downstream. $R_{2222}$ and $R_{3333}$ are stronger downstream of the chevron roots compared to downstream of chevron tips. This could be due to the fact that radial and azimuthal velocities become significant at the chevron roots. As the chevron tips block the jet flow, the flow tries to escape through the chevron roots and around the chevron slants. Therefore, both radial and azimuthal velocities become significant thereby intensifying $\mathrm{R}_{2222}$ and $\mathrm{R}_{3333}$ cross-correlations. Gaussian functions fit the $\mathrm{R}_{2222}, \mathrm{R}_{3333}, \mathrm{R}_{1212}$, $\mathrm{R}_{1313}$ and $\mathrm{R}_{2323}$ cross-correlations reasonably well (figures 21-24). Thus, Gaussian functions fit not only the dominant source cross-correlation, $\mathrm{R}_{1111}$, but also the other major source cross-correlations.

Chevron Roots

\begin{tabular}{ccc}
\multicolumn{3}{c}{ Chevron Tips } \\
\hline $\mathrm{x} / \mathrm{D}$ & $\mathrm{R}_{2222} / \mathrm{R}_{1111}$ & $\mathrm{R}_{3333} / \mathrm{R}_{1111}$ \\
\hline 2 & 0.47 & 0.49 \\
4 & 0.43 & 0.43 \\
6 & 0.42 & 0.36 \\
8 & 0.29 & 0.30 \\
10 & 0.32 & 0.30 \\
\hline
\end{tabular}

\begin{tabular}{ccc}
\hline $\mathrm{x} / \mathrm{D}$ & $\mathrm{R}_{2222} / \mathrm{R}_{1111}$ & $\mathrm{R}_{3333} / \mathrm{R}_{1111}$ \\
\hline 2 & 0.56 & 0.56 \\
4 & 0.49 & 0.49 \\
6 & 0.45 & 0.46 \\
8 & 0.33 & 0.36 \\
10 & 0.34 & 0.34 \\
\hline
\end{tabular}

Table 4: SMC006 - comparison of $R_{2222}$ and $R_{3333}$ with respect to the $R_{1111}$

\begin{tabular}{ccc}
\hline $\mathrm{x} / \mathrm{D}$ & $\mathrm{R}_{2222} / \mathrm{R}_{1111}$ & $\mathrm{R}_{3333} / \mathrm{R}_{1111}$ \\
\hline 2 & 0.37 & 0.35 \\
4 & 0.36 & 0.38 \\
6 & 0.42 & 0.43 \\
8 & 0.38 & 0.37 \\
10 & 0.30 & 0.27 \\
\hline
\end{tabular}

Table 5: SMC000 - comparison of $\mathrm{R}_{2222}$ and $\mathrm{R}_{3333}$ with respect to the $\mathrm{R}_{1111}$

We have already shown that RANS scales multiplied by the proportionality constants, $\sqrt{\mathrm{C}_{1111}}, \mathrm{C}_{l i}$ and $\mathrm{C}_{\tau}$ give a good fit to $\mathrm{R}_{1111}(\mathbf{x}, \xi, \tau)$ calculated from the LES results. Now, we investigate the other significant components of $\mathrm{R}_{i j k l}$. Figures 25-30 confirm that the shape of the $\mathrm{R}_{1111}, \mathrm{R}_{2222}$ and $\mathrm{R}_{3333}$ cross-correlations based on RANS scales (using the same proportionality constants as for the $\mathrm{R}_{1111}$ ). They show a reasonably good agreement with the Gaussian fit to LES flowfield (using equation 9). This further supports the fact that RANS scales that are multiplied by the proportionality constants can indeed define the acoustic sources accurately. 


\section{IV.C. Noise Propagation}

In the previous section, the major source cross-correlations were identified and we showed that they can be described by the RANS amplitude, length and time scales that are scaled by the determined proportionality constants: $\sqrt{\mathrm{C}_{1111}}=0.25, \mathrm{C}_{l 1}=0.26, \mathrm{C}_{l 2}=0.08, \mathrm{C}_{l 3}$ $=0.08$ and $\mathrm{C}_{\tau}=0.14$. The relative magnitudes of $\mathrm{R}_{i j k l}(\mathbf{x}, \mathbf{0}, 0)$ cross-correlations with respect to the $\mathrm{R}_{1111}(\mathbf{x}, \mathbf{0}, 0)$ cross-correlation are taken from the LES flowfield (shown in figure 20). The noise propagation from these sources to the jet far-field is determined through the calculation of a Green's function. The Green's function is the solution of the adjoint Linearised Euler Equations (LEEs). With a numerical solution, it can include the effects of scattering by the nozzle and the axial development of the jet mean flow (Karabasov et al. ${ }^{16}$ ). Instead for simplicity, we use a local parallel jet flow approximation. Then the Green's function can be quickly calculated analytically (Tam and Aurialt ${ }^{18}$ ).

\section{IV.C.1. Sound Power Spectral Density}

The sound power spectral density (PSD) is calculated at $30^{\circ}$ and $90^{\circ}$ to the jet axis. The reason for choosing these two particular angles is that the far-field noise at $30^{0}$ is affected significantly by the jet mean flow (Ffowcs Williams, ${ }^{27}$ Dowling et al. ${ }^{23}$ Karabasov et al. ${ }^{28}$ ), whereas that at $90^{\circ}$ is not. The PSD predictions by our modelling approach is compared with NASA Small Hot Jet Acoustic Rig (SHJAR) measurements conducted by Bridges and Brown $^{1}$ at a distance of 40 jet diameters from the nozzle exit. There is excellent agreement between the far-field noise predictions and measurements (figures 31 and 32). They match within $2-3 \mathrm{~dB}$ at both $30^{\circ}$ and $90^{\circ}$ to the jet axis for $0.02 \leq \mathrm{St} \leq 2$. The peak Strouhal number at both angles is approximately 0.2 i.e. peak frequency $=2 \mathrm{kHz}$. The spectral shape has a sharper maximum and a faster roll-off at $30^{\circ}$, whereas it is flat and broad at $90^{\circ}$ to the jet axis. These spectral shapes are similar for both the chevron and round jets. Both the peak Strouhal number and the spectral shape are very well captured in the predictions. At $30^{0}$ to the jet axis, the chevron jet has significantly reduced low-frequency noise compared to the round jet i.e. for $\mathrm{St} \leq 0.2$, the far-field noise is reduced by $5-6 \mathrm{~dB}$ (figure 31). This demonstrates the strong impact of the chevron nozzle on jet noise reduction, particularly at low-frequencies. However, there is no benefit at high-frequencies. At $90^{0}$ to the jet axis, the chevron jet has considerably reduced low-frequency noise compared to the round jet i.e. for St $\leq 0.2$, the far-field noise is reduced by $2-3 \mathrm{~dB}$ (figure 32 ). This indicates that the chevron jet has significantly reduced low-frequency noise for a wide range of radiation angles. However, it has slightly increased high-frequency noise compared to the round jet. This increase in the high-frequency noise is due to enhanced turbulence intensity by chevrons close to the nozzle exit. 


\section{IV.C.2. Overall Sound Pressure Level}

Figure 33 shows that there is a good agreement between predictions and measurements for the overall sound pressure level directivity. The overall sound pressure level is predicted very well in the peak noise direction by the LES-based modelling approach whereas, there is a slight over-prediction by the RANS-based modelling approach. At large angles to the jet axis, the overall sound pressure level is very well predicted by the RANS-based modelling approach whereas, it is under-predicted by the LES-based modelling approach. Overall, the RANS-based modelling approach predicts the overall sound pressure level very well at high angles and reasonably well at low angles to the jet axis.

\section{IV.C.3. Comparison of RANS-based and LES-based Modelling Approaches}

Figure 34 shows that the RANS-based modelling approach predicts far-field noise better than the Ffowcs Williams-Hawkings $(\mathrm{FW}-\mathrm{H})$ approach, particularly at high frequencies. Xia et al. ${ }^{6}$ used the same LES results to predict the far-field noise using the FW-H approach. There is some difficulty in closing the FW-H control surface when calculating the far-field noise emanated from a jet. However, there are some discrepancies between the far-field noise predictions and Bridges and Brown ${ }^{1}$ measurements at low-frequencies.

\section{IV.C.4. Effect of Anisotropy of Length Scales}

In Karabasov et al. ${ }^{16}$ modelling approach, the correlation length scales were assumed to be isotropic but in this study axial, radial and azimuthal length scales are calculated from the LES results. Figure 35 shows that the effect of anisotropy of length scales on the far-field noise predictions is around $3 \mathrm{~dB}$ and therefore it should be included in the source description for accurate far-field noise predictions.

\section{IV.C.5. Effect of Proportionality Constants}

Karabasov et $a l .{ }^{16}$ showed that the far-field noise changes within $0.2-0.7 \mathrm{~dB}$ by reducing the correlation length and time scales by $10 \%$. As the modelling approach in the present study also depends on the proportionality constants for radial and azimuthal length scales, the effect of these proportionality constants (for axial, radial and azimuthal length scales and a time scale) on the far-field noise is investigated. Figure 36 shows that there is a difference of $0.2-0.5 \mathrm{~dB}$ in the far-field noise predictions when the proportionality constants are varied by $15 \%$ from their original values. This confirms that the far-field noise predictions are not sensitive to small differences in the proportionality constants.

In table 6, the proportionality constants are compared with those of other researchers (Tam and Auriault, ${ }^{18}$ Morris and Farassat ${ }^{29}$ and Karabasov et al. ${ }^{16}$ ). The proportionality 


\begin{tabular}{cccc}
\hline & $\begin{array}{c}\text { Length scale } \\
\mathrm{C}_{l}\end{array}$ & $\begin{array}{c}\text { Time scale } \\
\mathrm{C}_{\tau}\end{array}$ & $\begin{array}{c}\text { Amplitude scale } \\
\sqrt{\mathrm{C}_{1111}}\end{array}$ \\
\hline Tam and Auriault $^{18}\left(\mathrm{M}_{j}=0.90\right)$ & 0.13 & 0.31 & 0.26 \\
Morris and Farassat $^{29}\left(\mathrm{M}_{j}=0.91\right)$ & 0.78 & 1.00 & 0.26 \\
Karabasov et al. $^{16}\left(\mathrm{M}_{j}=0.75\right)$ & 0.37 & 0.36 & 0.25 \\
Current prediction $\left(\mathrm{M}_{j}=0.90\right)$ & $0.26,0.08,0.08$ & 0.14 & 0.25 \\
\hline
\end{tabular}

Table 6: Comparison of the proportionality constants for amplitude, length and time scales

constant for the amplitude scale, $\sqrt{\mathrm{C}_{1111}}$, is around 0.25 for other researchers as well. The proportionality constants for the length and time scales are all of the same order of magnitude although some differences are seen.

\section{IV.C.6. Effect of Major Cross-correlations}

Figure 37 shows the contributions from the major cross-correlations to the jet noise. The $\mathrm{R}_{1212}, \mathrm{R}_{1313}$ and other cross-correlations equal to them by symmetry have the largest contribution to the jet noise at $30^{0}$ to the jet axis whereas, the $R_{2323}$ cross-correlation has the largest contribution to the jet noise at $90^{\circ}$ to the jet axis (figure 37 ). At $30^{0}$ to the jet axis, the low-frequency noise is captured very well just by including the contributions from $\mathrm{R}_{1212}$, $\mathrm{R}_{1313}$ and other cross-correlations equal to them by symmetry. At $90^{0}$ to the jet axis, the $\mathrm{R}_{2323}$ cross-correlation has the largest contribution to the jet noise over the entire frequency range.

\section{IV.C.7. Effect of Turbulence Models}

The standard $k-\epsilon$ turbulence model has been used so far for the RANS flow calculations. To examine the effect of turbulence models on far-field noise predictions, various turbulence models such as RNG and realisable $k-\epsilon$, standard and SST $k-\omega$, Reynolds Stress models were used. For the comparison of turbulence models, the SMC006 chevron jet is used as the baseline. The RANS scales based on $k-\epsilon$ turbulence models are defined as: amplitude scale $=(2 \bar{\rho} k)^{2} ;$ time scale $=k / \epsilon$ and length scale $=k^{3 / 2} / \epsilon$. The RANS scales based on $k-\omega$ turbulence models are defined as: amplitude scale $=(2 \bar{\rho} k)^{2}$; time scale $=1 /(0.09 \omega)$ and length scale $=k^{1 / 2} / \omega$ where $k$ is turbulent kinetic energy; $\epsilon$ is turbulent dissipation rate and $\omega$ is turbulent eddy frequency $=0.09 \epsilon / k$ (Launder and Spalding ${ }^{21}$ ).

To confirm that the constants of proportionality hold good for various turbulence models, the derived RANS scales were validated against the LES scales. Figure 38 confirms that the constants of proportionality for amplitude, length and time scales hold good for all the turbulence models. Although some differences are seen in these scales, the crucial question 
is whether these are large enough to affect the far-field noise predictions. With the same proportionality constants, figure 39 shows that there is a good agreement between the farfield noise predictions and Bridges and Brown ${ }^{1}$ measurements. The effect of turbulence models on far-field noise predictions is less than $0.5 \mathrm{~dB}$. These results indicate that the predicted far-field noise is almost insensitive to the turbulence model.

\section{Summary and Conclusions}

From the fourth-order space-time cross-correlations calculated from the LES flowfield, the correlation length scales are found not to be the same for separation distances in different directions i.e. the axial length scale is 3-4 times the radial and azimuthal length scales and therefore the anisotropy of correlation length scales is considered in our modelling approach. Although $\mathrm{R}_{1111}$, the cross-correlation based on axial velocity fluctuations, is the dominant component of the source cross-correlation function, there is a considerable contribution from other cross-correlations such as $\mathrm{R}_{2222}, \mathrm{R}_{3333}, \mathrm{R}_{1212}, \mathrm{R}_{1313}$ and $\mathrm{R}_{2323}$ and other components equal to them by symmetry. To account for the anisotropy of the acoustic sources, the relative magnitude of the cross-correlations, $\mathrm{R}_{i j k l}(\mathbf{x}, \mathbf{0}, 0)$, with respect to the $\mathrm{R}_{1111}(\mathbf{x}, \mathbf{0}, 0)$ is taken from the LES for an accurate description of the acoustic source. All the major source cross-correlations have the shape of Gaussian functions with the same length and time scales. Within 4 jet diameters from the nozzle exit, the chevron jet intensifies both $R_{2222}$ and $R_{3333}$ relative to their values for the round jet. The cross-correlations rapidly decay with axial distance for the chevron jet, whereas they remain almost constant for the first 10 jet diameters for the round jet. Hence, the noise sources are concentrated close to the nozzle for the chevron jet while they have greater axial spread for the round jet. The chevron jet weakens the dominant source component, the $\mathrm{R}_{1111}$ cross-correlation, by $50-60 \%$ compared to that of the round jet.

The RANS captures well the variation of amplitude, length and time scales of the crosscorrelations with both source position and nozzle geometry. The RANS scales multiplied by the proportionality constants, $\sqrt{\mathrm{C}_{1111}}=0.25, \mathrm{C}_{l 1}=0.26, \mathrm{C}_{l 2}=0.08, \mathrm{C}_{l 3}=0.08$ and $\mathrm{C}_{\tau}=0.14$, can be used for an accurate description of the acoustic sources instead of the LES scales. These proportionality constants are found to be independent of source position within the jet and they are quite close for chevron and round jets. With the acoustic sources described by RANS scales that are scaled by the proportionality constants and the relative magnitudes of the $\mathrm{R}_{i j k l}(\mathbf{x}, \mathbf{0}, 0)$ cross-correlations with respect to the $\mathrm{R}_{1111}(\mathbf{x}, \mathbf{0}, 0)$ crosscorrelation are taken from LES, the far-field noise predictions showed excellent agreement with the measurements conducted by Bridges and Brown ${ }^{1}$ for a wide range of frequencies and observer angles. 
The effect of the major cross-correlations in terms of their contribution to jet noise is investigated. $\mathrm{R}_{1212}, \mathrm{R}_{1313}$ and other cross-correlations equal to them by symmetry have the largest contribution to the jet noise at $30^{\circ}$ to the jet axis whereas, the $\mathrm{R}_{2323}$ cross-correlation has the largest contribution to the jet noise at $90^{\circ}$ to the jet axis. This trend is observed in both round and chevron jets. At $30^{\circ}$ to the jet axis, the low-frequency noise is captured very well just by including the contribution from $\mathrm{R}_{1212}, \mathrm{R}_{1313}$ and other cross-correlations equal to them by symmetry. At $90^{\circ}$ to the jet axis, the far-field noise is captured very well over the entire frequency range just by including the contribution from the $\mathrm{R}_{2323}$ cross-correlation.

In Karabasov et al. ${ }^{16}$ modelling approach, only the axial length scale was considered but in this study axial, radial and azimuthal length scales are considered in the source description. It was found that the effect of anisotropy of length scales on the far-field noise predictions is around $3 \mathrm{~dB}$ and hence it should be considered for accurate source description and far-field noise predictions. As the modelling approach also depends on the proportionality constants for length (axial, radial and azimuthal) and time scales, the effect of these proportionality constants on the far-field noise is investigated. It was found that there is a difference of $0.2-0.5 \mathrm{~dB}$ in the far-field noise predictions when the proportionality constants are varied by about $15 \%$ from their original values. This confirms that the far-field noise predictions are not sensitive to small differences in the proportionality constants.

The standard $k-\epsilon$ turbulence model is used for RANS flow calculations. To examine the effect of turbulence models on far-field noise predictions, various turbulence models such as RNG and realisable $k-\epsilon$, standard and SST $k-\omega$, Reynolds Stress models are used. The same proportionality constants are found to give a reasonable approximation to the LES scales for all the turbulence models. Moreover with the same proportionality constants, there is an excellent agreement between the far-field noise predictions and measurements. The effect of turbulence models on far-field noise predictions is found to be less than $0.5 \mathrm{~dB}$. This shows that the far-field noise prediction is almost insensitive to the turbulence model.

To conclude, the results indicate that the modelling approach is capable of assessing advanced noise-reduction concepts and could contribute to the development of quieter nozzles for future civil aircraft.

\section{Acknowledgements}

Mr Depuru Mohan expresses his sincere gratitude to St John's College, University of Cambridge, UK, for the award of a Dr Manmohan Singh Scholarship and Cambridge Commonwealth, European and International Trust for the award of an Honorary Cambridge International Scholarship. Dr Karabasov wishes to thank the Royal Society of London, UK, for the award of University Research Fellowship. Dr Xia acknowledges the computational 
time on European High Performance Computing (HPC) systems, PRACE, under the project 2010PA0649. Authors are grateful to Drs Bridges, Brown, Georgiadis and DeBonis, NASA Glenn Research Centre, USA, for providing the experimental data.

\section{References}

${ }^{1}$ Bridges, J. and Brown, C., "Parametric testing of chevrons on single flow hot jets," NASA Technical Report, 2004, pp. 1-17.

${ }^{2}$ Gudmundsson, K. and Colonius, T., "Linear stability analysis of chevron jet profiles," Bulletin of the American Physical Society, Vol. 52, 2007.

${ }^{3}$ Callender, B., Gutmark, E., and Martens, S., "Far-field acoustic investigation into chevron nozzle mechanisms and trends," AIAA journal, Vol. 43, No. 1, 2005, pp. 87-95.

${ }^{4}$ Callender, B., Gutmark, E., and Martens, S., "Near-field investigation of chevron nozzle mechanisms," AIAA Journal, Vol. 46, No. 1, 2008, pp. 36-45.

${ }^{5}$ Tide, P. and Srinivasan, K., "Novel chevron nozzle concepts for jet noise reduction," Proceedings of the Institution of Mechanical Engineers, Part G: Journal of Aerospace Engineering, Vol. 223, No. 1, 2009, pp. $51-67$.

${ }^{6}$ Xia, H. and Tucker, P., "Numerical simulation of single-stream jets from a serrated nozzle," Flow, Turbulence and Combustion, Vol. 88, 2012, pp. 3-18.

${ }^{7}$ Engel, R., Silva, C., and Deschamps, C., "Application of RANS-based method to predict acoustic noise of chevron nozzles," Applied Acoustics, Vol. 79, 2014, pp. 153-163.

${ }^{8}$ Balsa, T., Gliebe, P., Kantola, R., Mani, R., Stringas, E., and Wang, J., "High velocity jet noise source location and reduction. Task 2 - theoretical developments and basic experiments." Federal Aviation Administration, Vol. 2, No. FAA-RD-76-79, 1978.

${ }^{9}$ Khavaran, A., Krejsa, E., and Kim, C., "Computation of supersonic jet mixing noise for an axisymmetric convergent-divergent nozzle," Journal of Aircraft, Vol. 31, No. 3, 1994, pp. 603-609.

${ }^{10}$ Khavaran, A., "Role of anisotropy in turbulent mixing layer," AIAA Journal, Vol. 37, No. 7, 1999, pp. $832-841$.

${ }^{11}$ Viswanathan, K., "Assessment of jet noise theory/prediction methods," Proceedings of the Jet Noise Workshop, NASA-CP 2001-211152, 2001, pp. 979-1010.

${ }^{12}$ Tam, C. and Auriault, L., "Jet mixing noise from fine-scale turbulence," AIAA Journal, Vol. 37, No. 2, 1999, pp. 145-153.

${ }^{13}$ Freund, J. B., Samanta, A., Wei, M., and Lele, S., "The robustness of acoustic analogies," 11th AIAA/CEAS Aeroacoutics Conference, Vol. 2940, 2005, pp. 1-8.

${ }^{14}$ Wang, M., Freund, J. B., and Lele, S. K., "Computational prediction of flow-generated sound," Annual Review of Fluid Mechanics, Vol. 38, 2006, pp. 483-512.

${ }^{15}$ Goldstein, M. and Leib, S., "The aeroacoustics of slowly diverging supersonic jets," Journal of Fluid Mechanics, Vol. 600, No. 1, 2008, pp. 291-337.

${ }^{16}$ Karabasov, S., Afsar, M., Hynes, T., Dowling, A., McMullan, W., Pokora, C., Page, G., and McGuirk, J., "Jet noise: acoustic analogy informed by large eddy simulation," AIAA Journal, Vol. 48, No. 7, 2010, pp. $1312-1325$. 
${ }^{17}$ Goldstein, M., "A generalized acoustic analogy," Journal of Fluid Mechanics, Vol. 488, 2003, pp. 315333.

${ }^{18}$ Tam, C. and Auriault, L., "Mean flow refraction effects on sound radiated from localized sources in a jet," Journal of Fluid Mechanics, Vol. 370, No. 2, 1998, pp. 149-174.

${ }^{19}$ Xia, H., Karabasov, S., Graham, O., Tucker, P., Dowling, A., Depuru Mohan, N., and Hynes, T., "Hybrid RANS-LES modeling of chevron nozzles with prediction of far field sound," AIAA Aerospace Sciences Meeting, 2011.

${ }^{20}$ Dowling, A. and Hynes, T., "Sound generation by turbulence," European Journal of MechanicsB/Fluids, Vol. 23, No. 3, 2004, pp. 491-500.

${ }^{21}$ Launder, B. and Spalding, D., "The numerical computation of turbulent flows," Computer methods in applied mechanics and engineering, Vol. 3, No. 2, 1974, pp. 269-289.

${ }^{22}$ Agarwal, A., Morris, P., and Mani, R., "Calculation of sound propagation in nonuniform flows: suppression of instability waves," AIAA Journal, Vol. 42, No. 1, 2004, pp. 80-88.

${ }^{23}$ Dowling, A., Ffowcs Williams, J., and Goldstein, M., "Sound production in a moving stream," Philosophical Transactions of the Royal Society of London. Series A, Mathematical and Physical Sciences, Vol. 288, No. 1353, 1978, pp. 321-349.

${ }^{24}$ Karabasov, S. and Hynes, T., "Adjoint linearized Euler solver in the frequency domain for jet noise modeling," 12th AIAA/CEAS Aeroacoustics Conference, Vol. 2673, 2006.

${ }^{25}$ Lighthill, M., "On sound generated aerodynamically. I. General theory," Proceedings of the Royal Society of London. Series A. Mathematical and Physical Sciences, Vol. 211, No. 1107, 1952, pp. $564-587$.

${ }^{26}$ Lighthill, M., "On sound generated aerodynamically. II. Turbulence as a source of sound," Proceedings of the Royal Society of London. Series A. Mathematical and Physical Sciences, Vol. 222, No. 1148, 1954, pp. 1-32.

${ }^{27}$ Ffowcs Williams, J., "The noise from turbulence convected at high speed," Philosophical Transactions of the Royal Society of London. Series A, Mathematical and Physical Sciences, Vol. 255, 1963, pp. 469-503.

${ }^{28}$ Karabasov, S., Hynes, T., and Dowling, A., "Effect of mean-flow evolution on sound propagation through non-uniform jet flows," AIAA Paper, Vol. 3655, 2007.

${ }^{29}$ Morris, P. and Farassat, F., "Acoustic analogy and alternate theories for jet noise prediction," $A I A A$ Journal, Vol. 40, No. 4, 2002, pp. 671-680. 


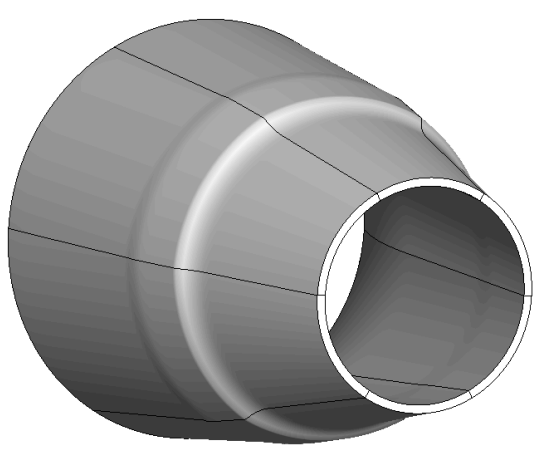

(a) $\mathrm{SMC000}$

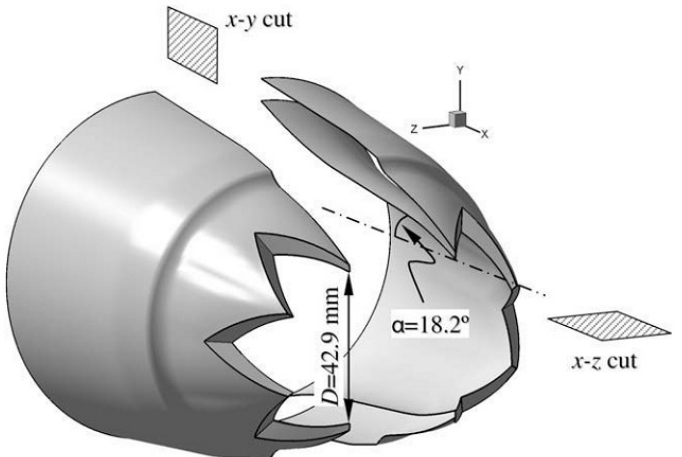

(b) $\mathrm{SMC006}$

Figure 1: The geometry of SMC000 and SMC006 nozzles (Bridges and Brown ${ }^{1}$ )

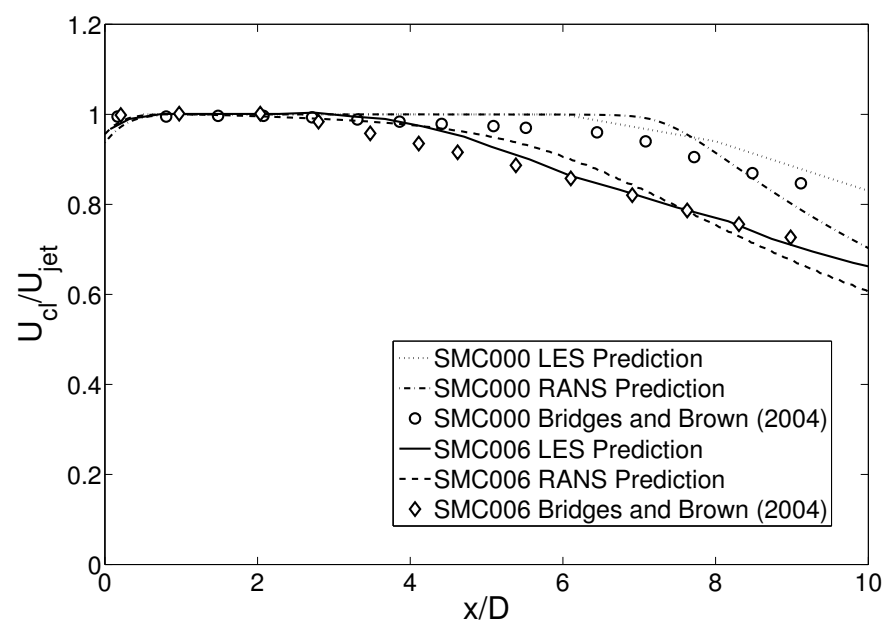

Figure 2: The decay of the jet centreline velocity

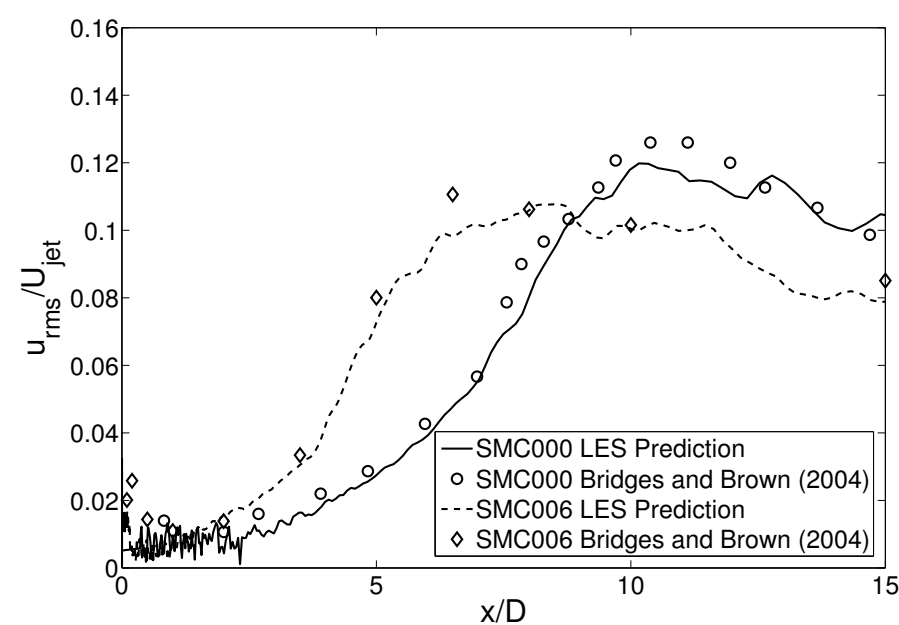

Figure 3: Turbulence intensity of the jet along its centreline 


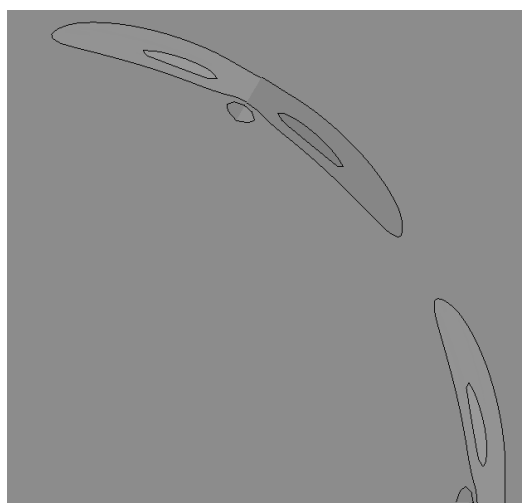

(a) $\mathrm{SMC000}$

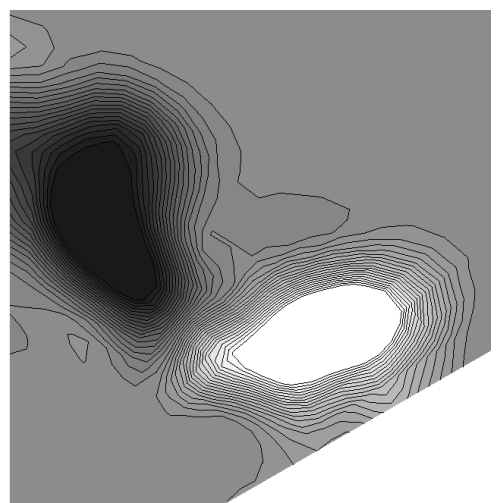

(b) $\mathrm{SMC006}$

Figure 4: Streamwise vorticity at $\mathrm{x} / \mathrm{D}=0.2:-7000 \mathrm{sec}^{-1}<\omega_{x}<+7000 \mathrm{sec}^{-1}$ (the streamwise vortices are usually formed around the nozzle lip, $\mathrm{r}=0.5 \mathrm{D}$ )

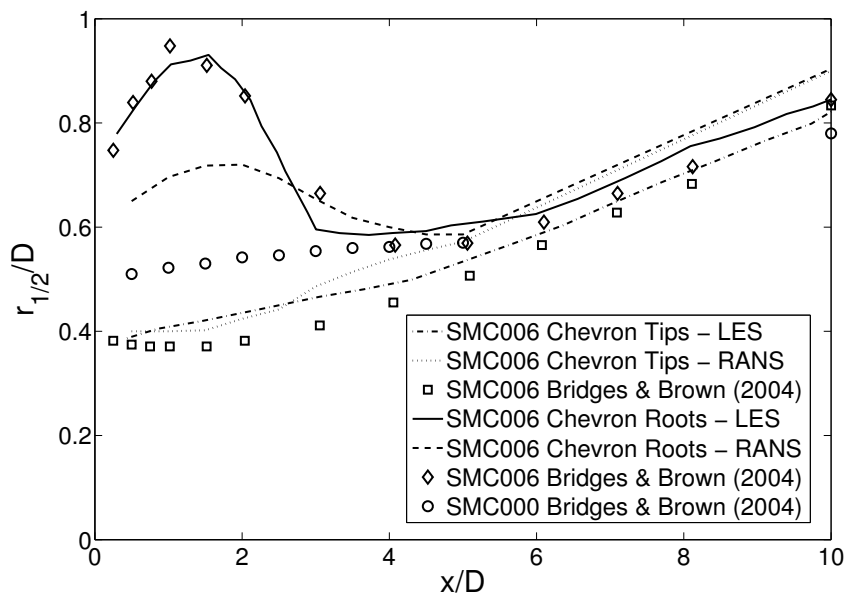

Figure 5: Spreading rate of the jet for $\mathrm{x} / \mathrm{D} \leq 10$

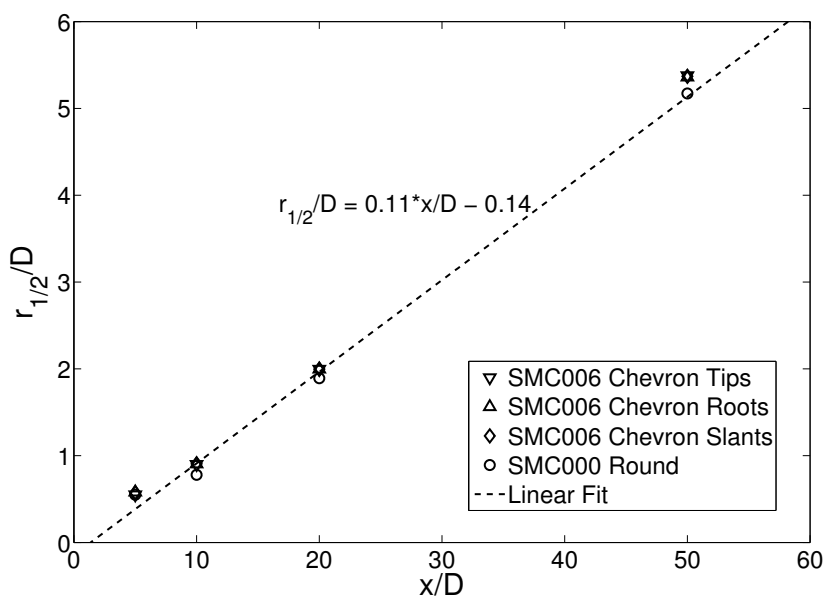

Figure 6: Spreading rate of the jet for $5 \leq \mathrm{x} / \mathrm{D} \leq 50$ 


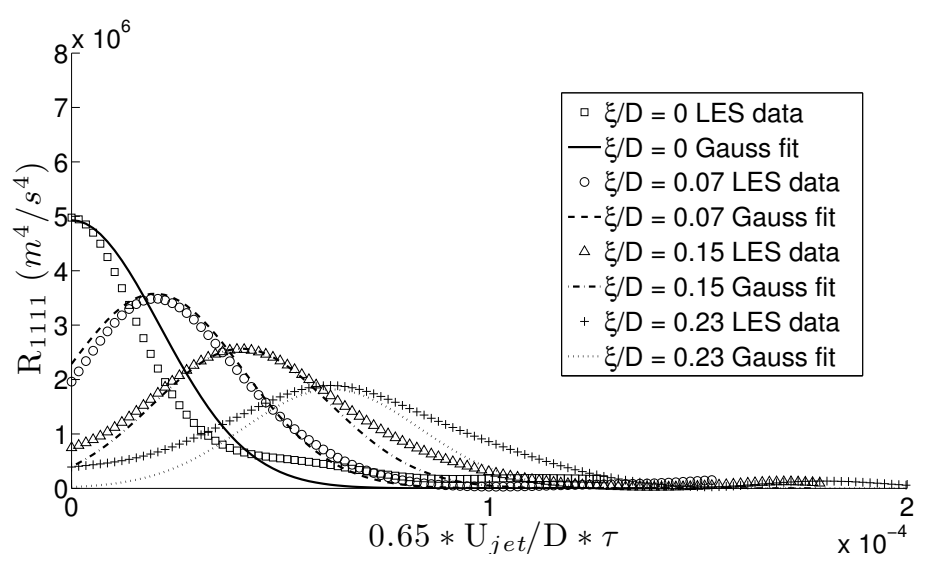

Figure 7: SMC006: $\mathrm{R}_{1111}$ cross-correlation $\left(\xi_{1} / \mathrm{D}\right.$ varies, $\xi_{2} / \mathrm{D}=0$ and $\left.\xi_{3} / \mathrm{D}=0\right)$ at $\mathrm{x} / \mathrm{D}=$ $4 ; \mathrm{r} / \mathrm{D}=0.5$ and $\theta=0^{0}$

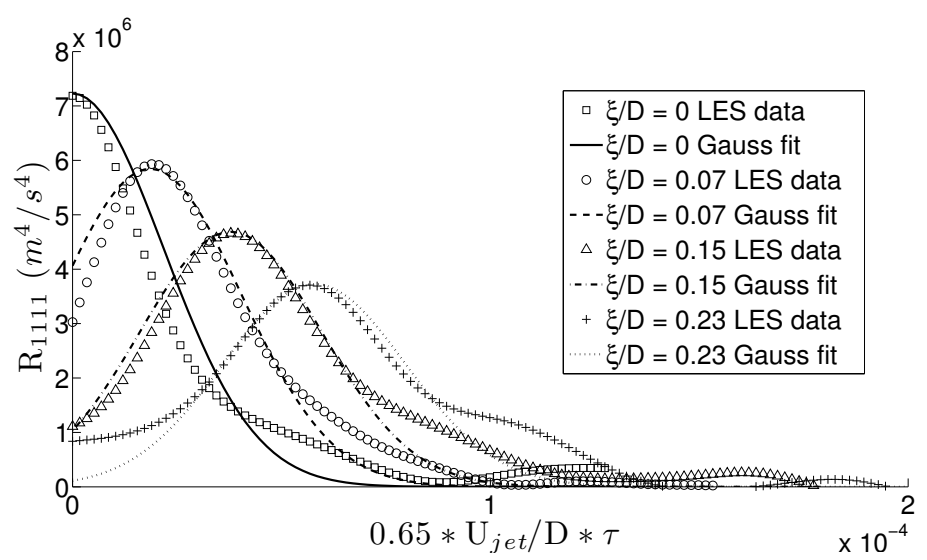

Figure 8: SMC000: $\mathrm{R}_{1111}$ cross-correlation $\left(\xi_{1} / \mathrm{D}\right.$ varies, $\xi_{2} / \mathrm{D}=0$ and $\left.\xi_{3} / \mathrm{D}=0\right)$ at $\mathrm{x} / \mathrm{D}=$ $4 ; \mathrm{r} / \mathrm{D}=0.5$ and $\theta=0^{0}$

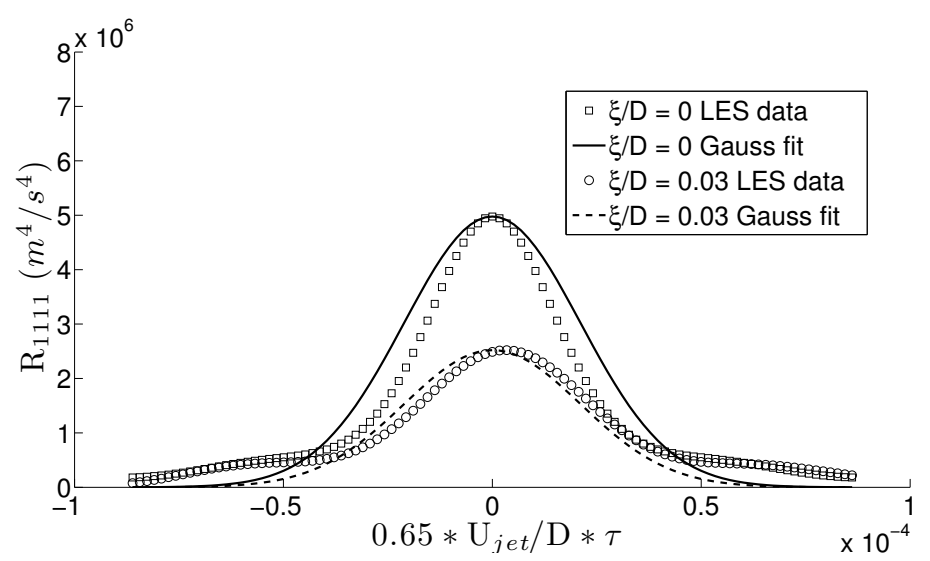

Figure 9: $\mathrm{SMC006}: \mathrm{R}_{1111}$ cross-correlation $\left(\xi_{1} / \mathrm{D}=0, \xi_{2} / \mathrm{D}\right.$ varies and $\left.\xi_{3} / \mathrm{D}=0\right)$ at $\mathrm{x} / \mathrm{D}=$ $4 ; \mathrm{r} / \mathrm{D}=0.5$ and $\theta=0^{0}$ 


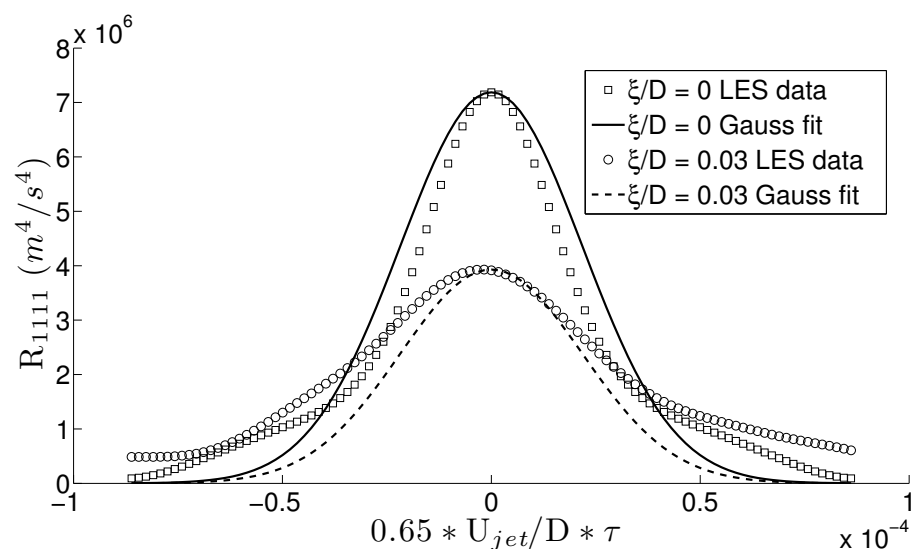

Figure 10: SMC000: $\mathrm{R}_{1111}$ cross-correlation $\left(\xi_{1} / \mathrm{D}=0, \xi_{2} / \mathrm{D}\right.$ varies and $\left.\xi_{3} / \mathrm{D}=0\right)$ at $\mathrm{x} / \mathrm{D}$ $=4 ; \mathrm{r} / \mathrm{D}=0.5$ and $\theta=0^{0}$

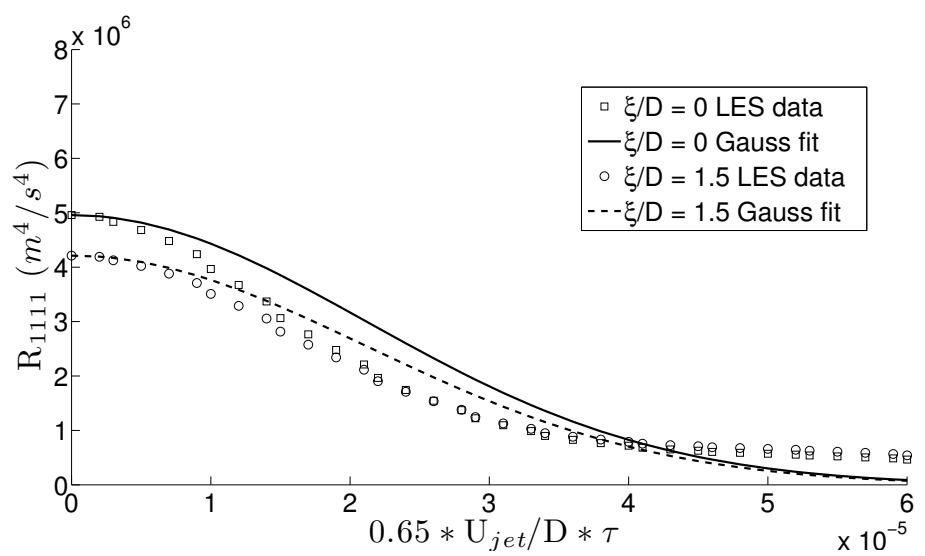

Figure 11: SMC006: $\mathrm{R}_{1111}$ cross-correlation $\left(\xi_{1} / \mathrm{D}=0, \xi_{2} / \mathrm{D}=0\right.$ and $\xi_{3} / \mathrm{D}$ varies $)$ at $\mathrm{x} / \mathrm{D}$ $=4 ; \mathrm{r} / \mathrm{D}=0.5$ and $\theta=0^{0}$

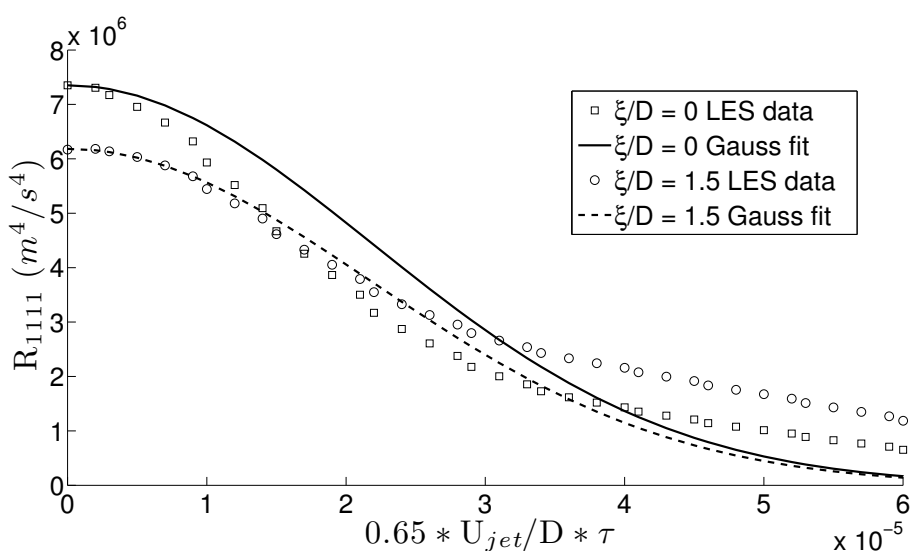

Figure 12: SMC000: $\mathrm{R}_{1111}$ cross-correlation $\left(\xi_{1} / \mathrm{D}=0, \xi_{2} / \mathrm{D}=0\right.$ and $\xi_{3} / \mathrm{D}$ varies $)$ at $\mathrm{x} / \mathrm{D}$ $=4 ; \mathrm{r} / \mathrm{D}=0.5$ and $\theta=0^{0}$ 


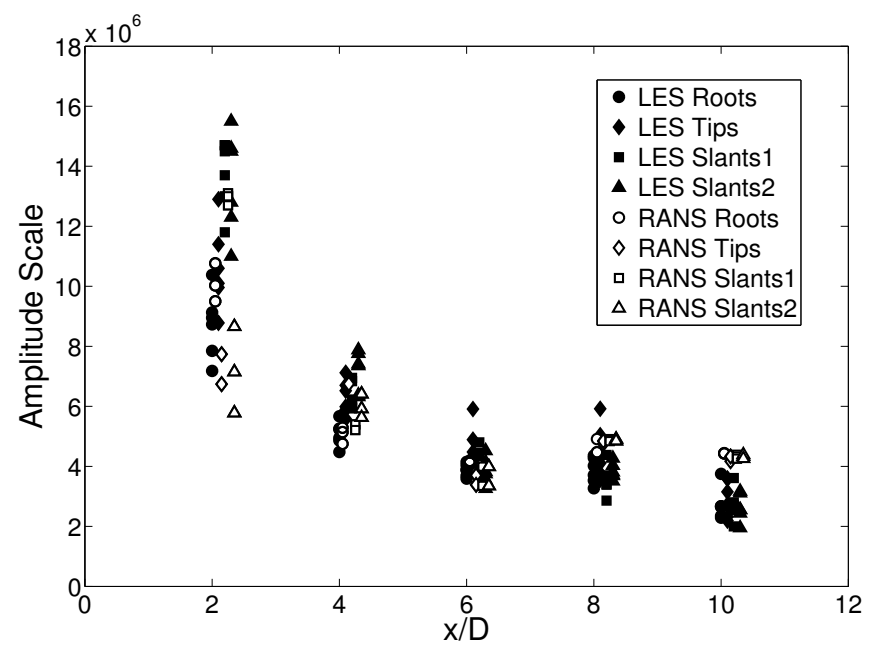

Figure 13: SMC006: Amplitude scale - Comparison of scaled-RANS and LES scales

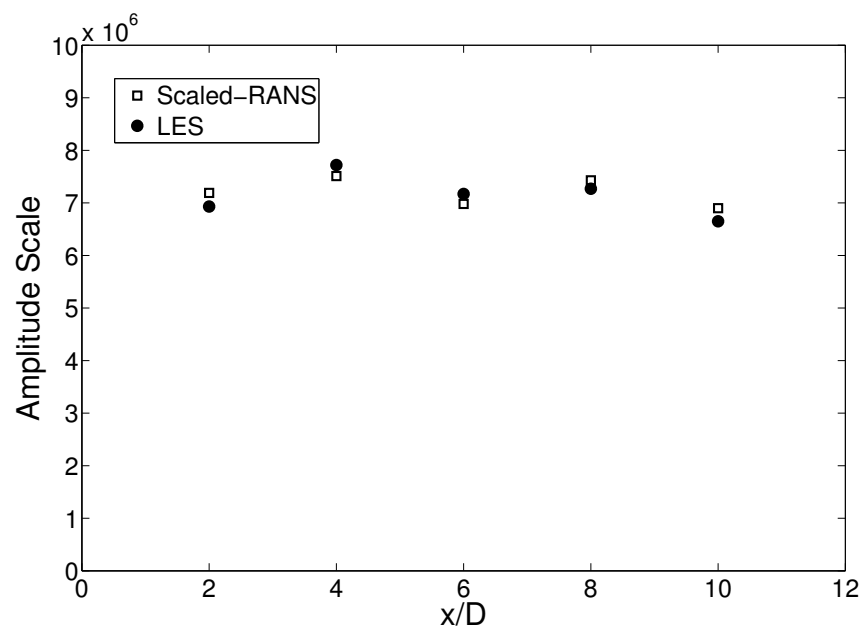

Figure 14: SMC000: Amplitude scale - Comparison of scaled-RANS and LES scales

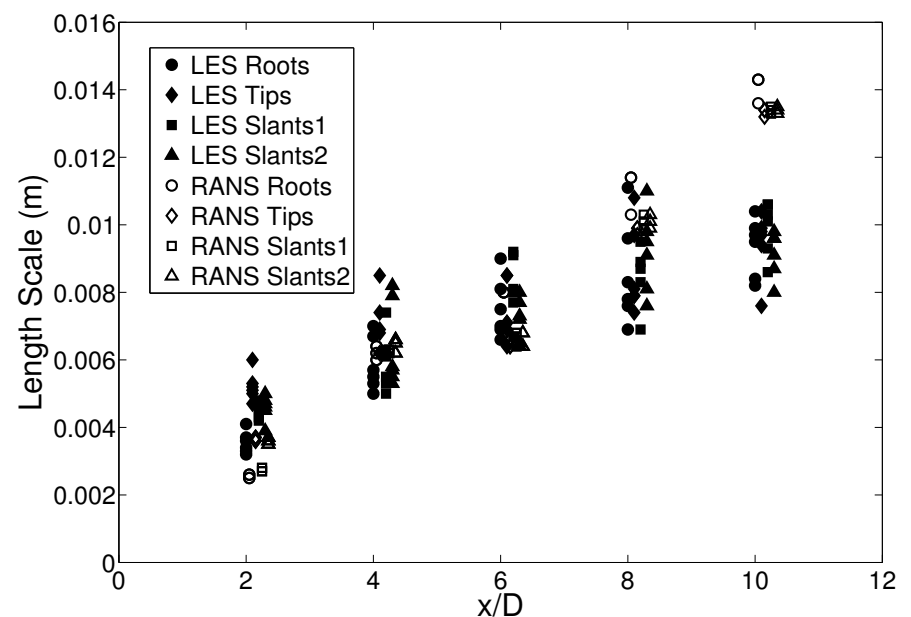

Figure 15: SMC006: Length scale - Comparison of scaled-RANS and LES scales 


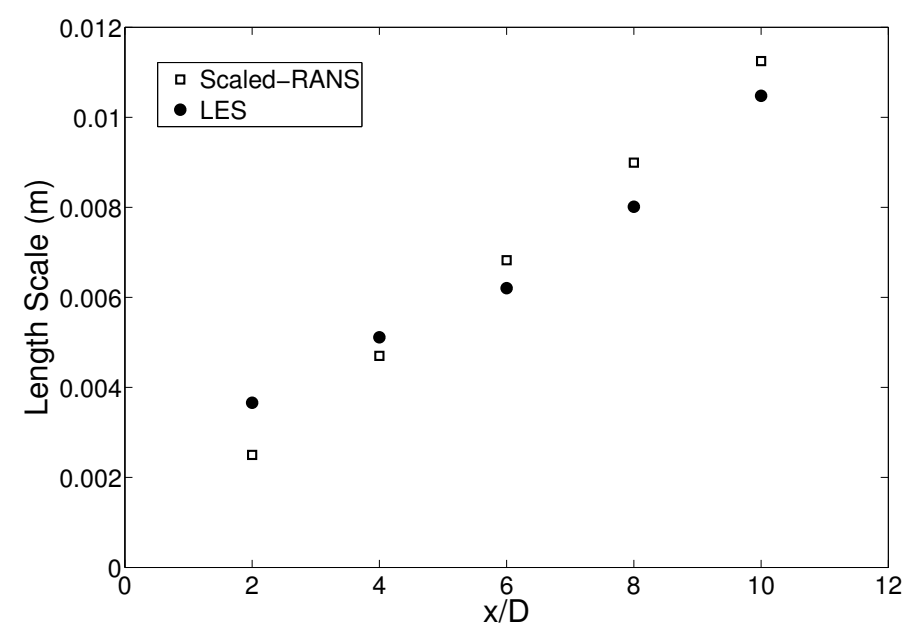

Figure 16: SMC000: Length scale - Comparison of scaled-RANS and LES scales

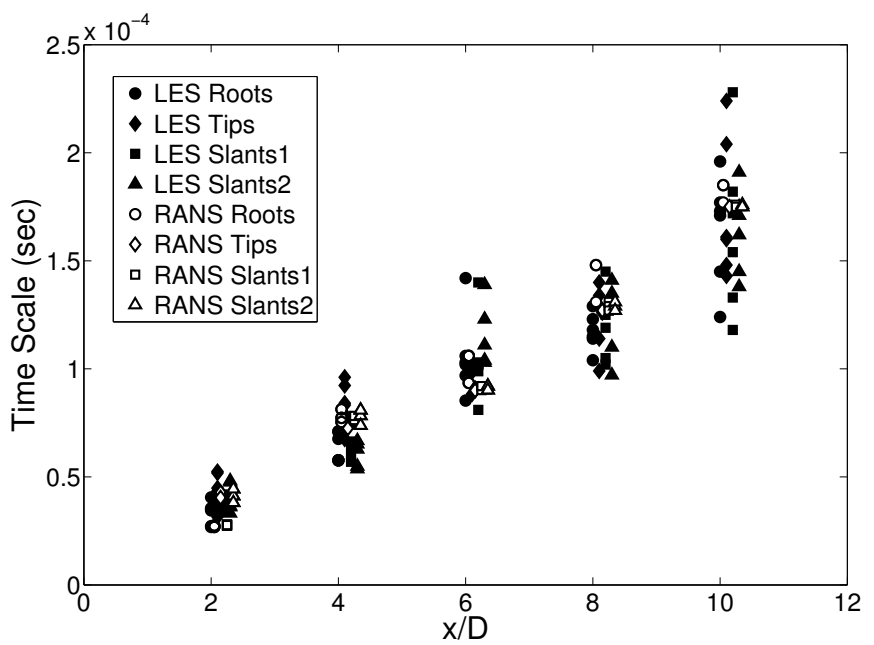

Figure 17: SMC006: Time scale - Comparison of scaled-RANS and LES scales

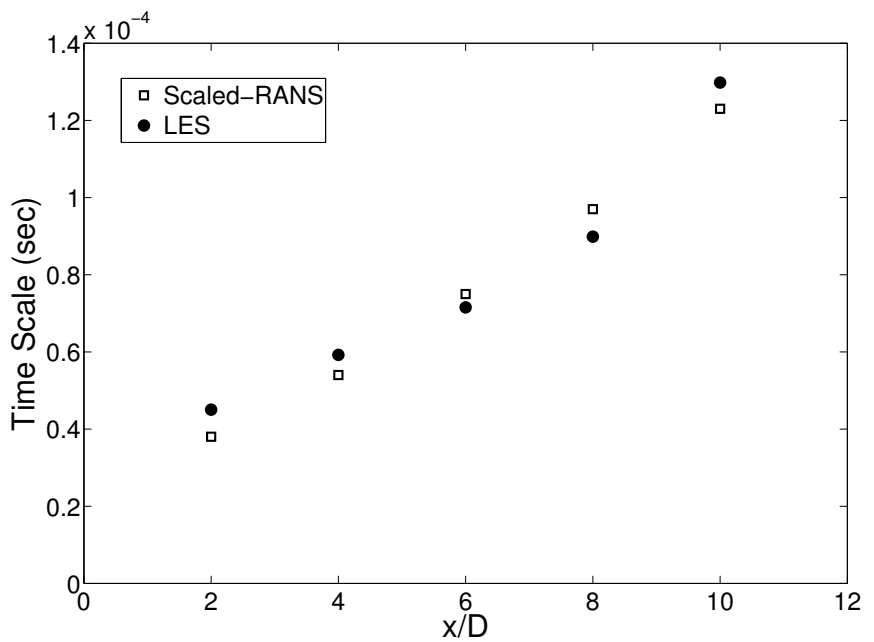

Figure 18: SMC000: Time scale - Comparison of scaled-RANS and LES scales 


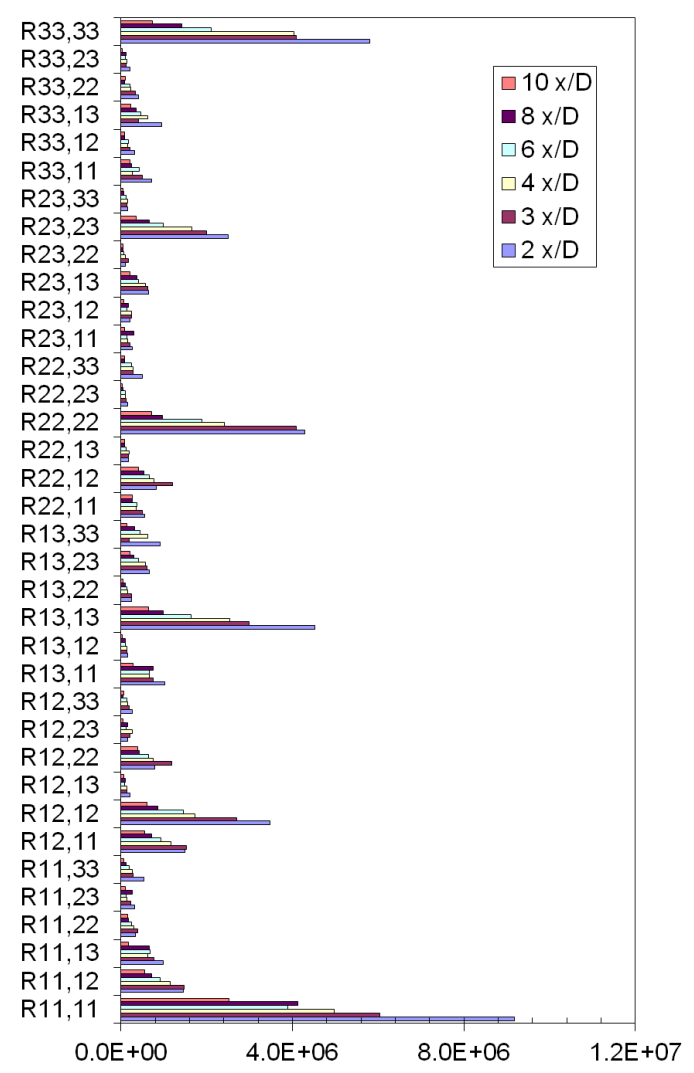

(a) $\mathrm{SMC006}$

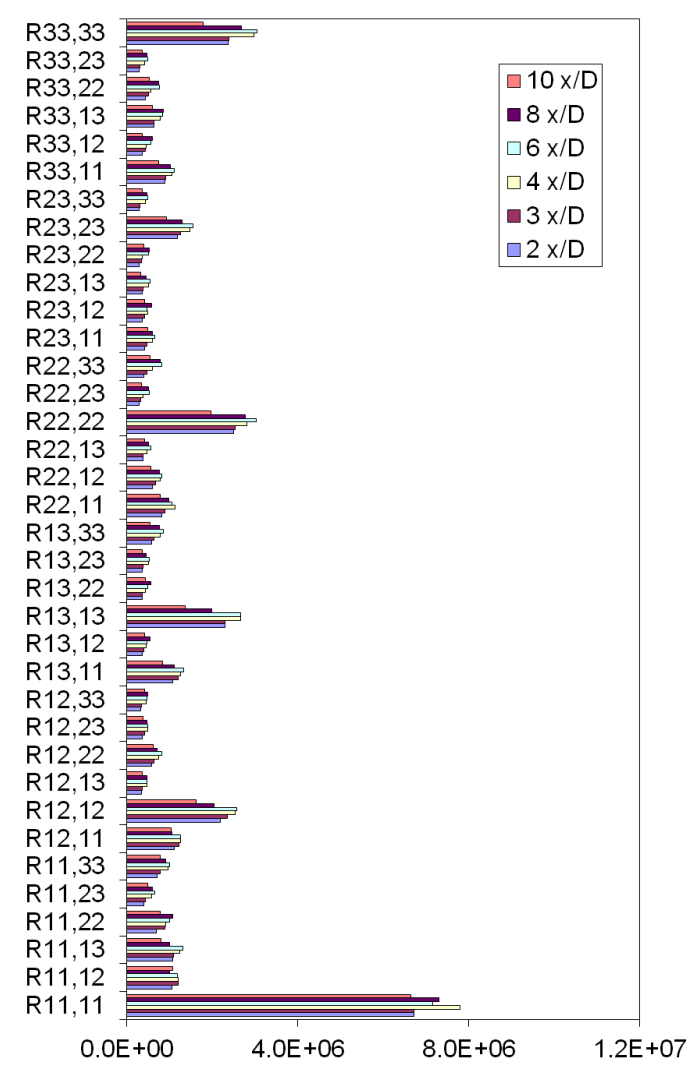

(b) SMC000

Figure 19: Absolute magnitudes of $\mathrm{R}_{i j k l}$ cross-correlations in $\mathrm{m}^{4} / \mathrm{s}^{4}$ 


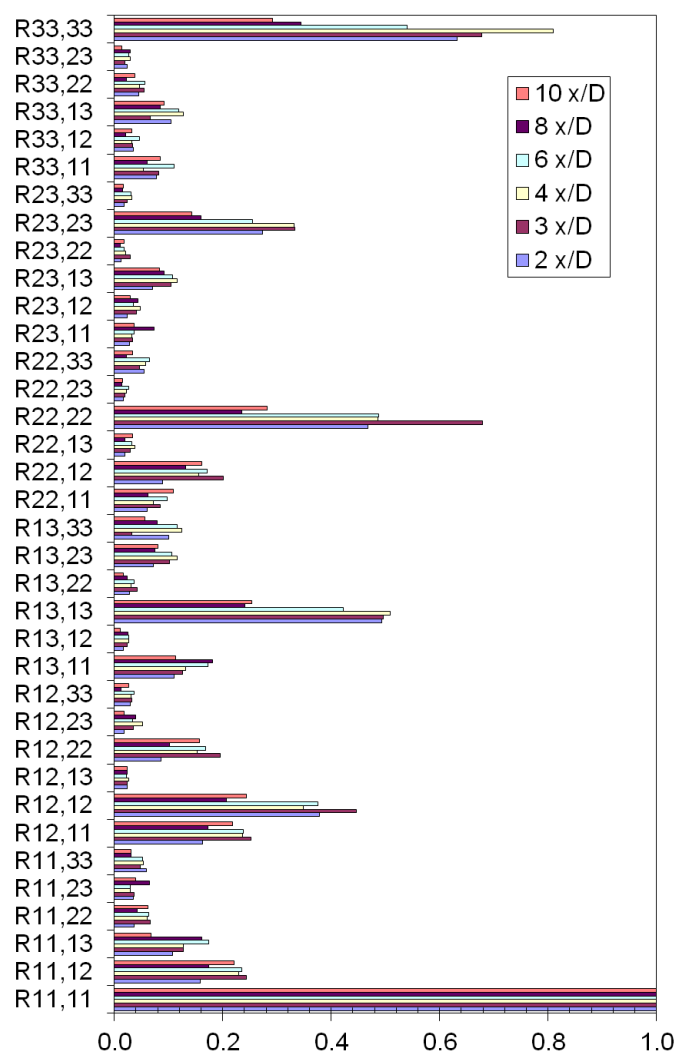

(a) $\mathrm{SMC006}$

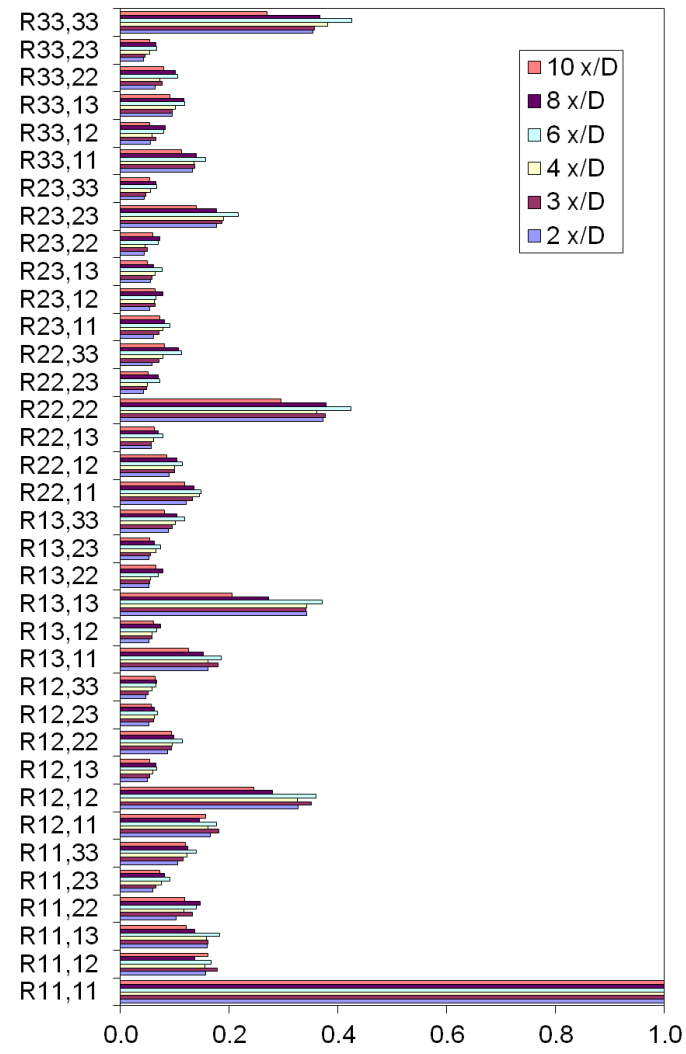

(b) $\mathrm{SMC000}$

Figure 20: Relative magnitudes of $\mathrm{R}_{i j k l}$ cross-correlations with respect to the $\mathrm{R}_{1111}$ crosscorrelation 


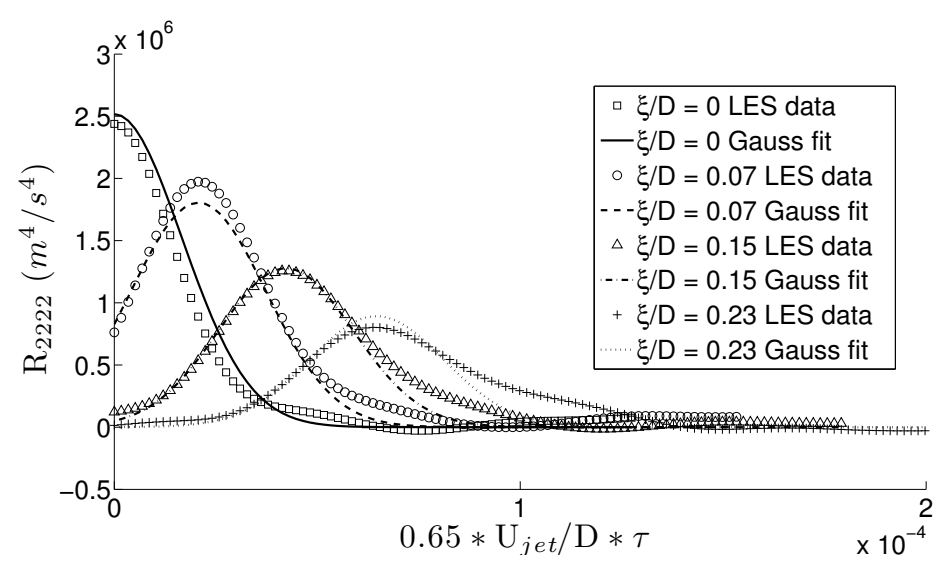

Figure 21: SMC006: $\mathrm{R}_{2222}$ cross-correlation $\left(\xi_{1} / \mathrm{D}=0, \xi_{2} / \mathrm{D}\right.$ varies and $\left.\xi_{3} / \mathrm{D}=0\right)$ at $\mathrm{x} / \mathrm{D}$ $=4 ; \mathrm{r} / \mathrm{D}=0.5$ and $\theta=0^{0}$

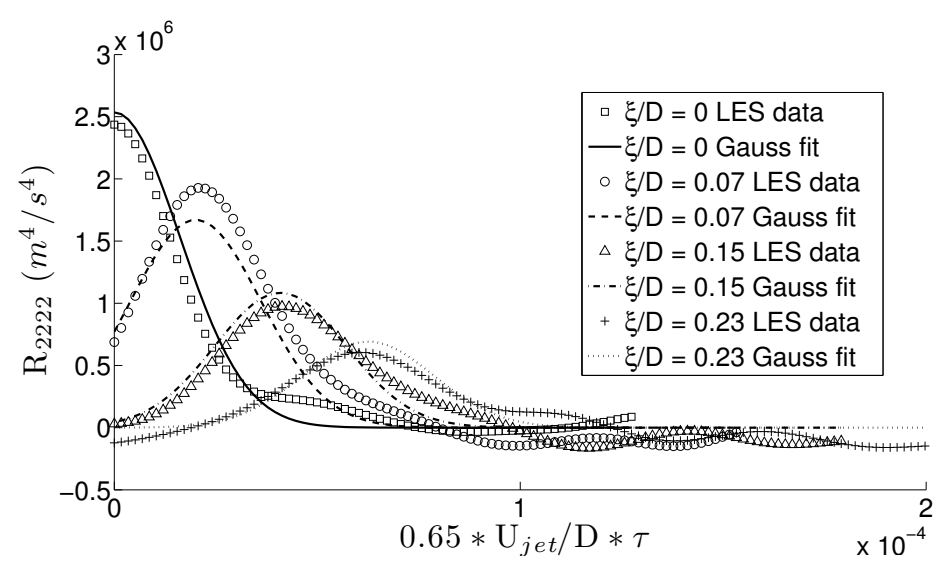

Figure 22: SMC000: $\mathrm{R}_{2222}$ cross-correlation $\left(\xi_{1} / \mathrm{D}=0, \xi_{2} / \mathrm{D}\right.$ varies and $\left.\xi_{3} / \mathrm{D}=0\right)$ at $\mathrm{x} / \mathrm{D}$ $=4 ; \mathrm{r} / \mathrm{D}=0.5$ and $\theta=0^{0}$

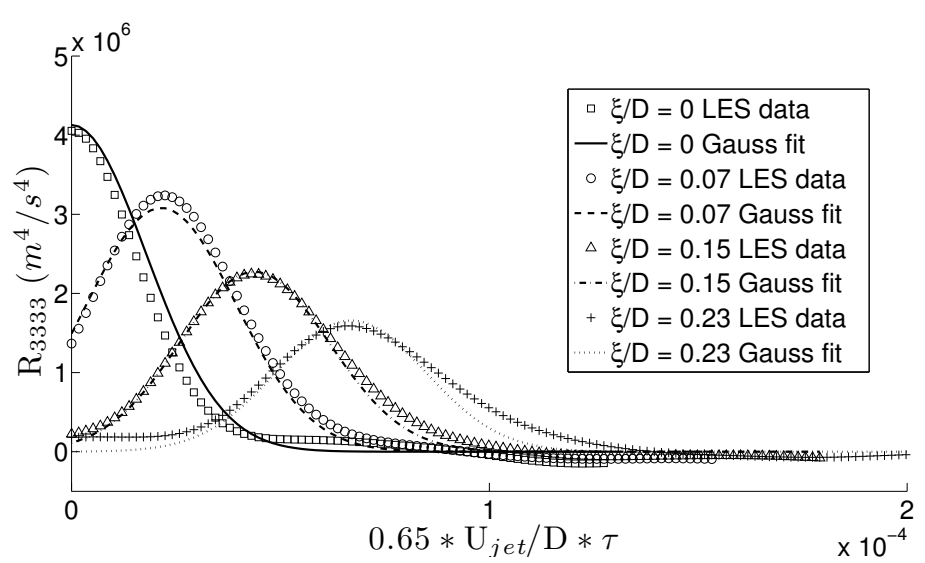

Figure 23: SMC006: $\mathrm{R}_{3333}$ cross-correlation $\left(\xi_{1} / \mathrm{D}=0, \xi_{2} / \mathrm{D}=0\right.$ and $\xi_{3} / \mathrm{D}$ varies $)$ at $\mathrm{x} / \mathrm{D}$ $=4 ; \mathrm{r} / \mathrm{D}=0.5$ and $\theta=0^{0}$ 


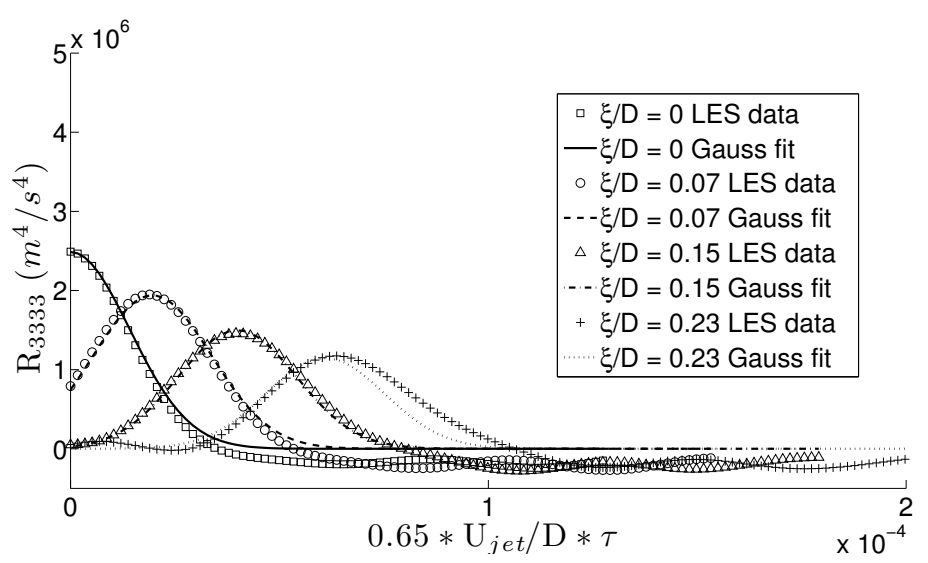

Figure 24: SMC000: $\mathrm{R}_{3333}$ cross-correlation $\left(\xi_{1} / \mathrm{D}=0, \xi_{2} / \mathrm{D}=0\right.$ and $\xi_{3} / \mathrm{D}$ varies $)$ at $\mathrm{x} / \mathrm{D}$ $=4 ; \mathrm{r} / \mathrm{D}=0.5$ and $\theta=0^{0}$

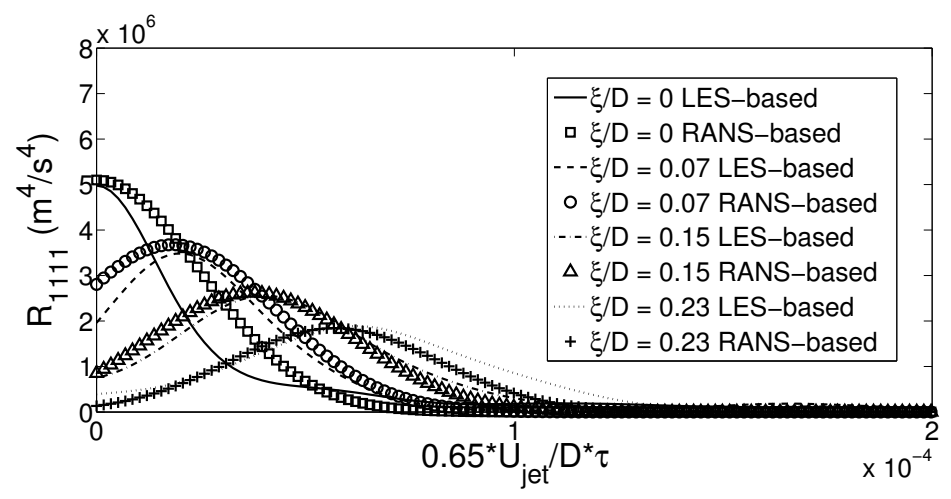

Figure 25: SMC006: $\mathrm{R}_{1111}$ cross-correlation $\left(\xi_{1} / \mathrm{D}\right.$ varies, $\xi_{2} / \mathrm{D}=0$ and $\left.\xi_{3} / \mathrm{D}=0\right)$ at $\mathrm{x} / \mathrm{D}$ $=4 ; \mathrm{r} / \mathrm{D}=0.5$ and $\theta=0^{0}$

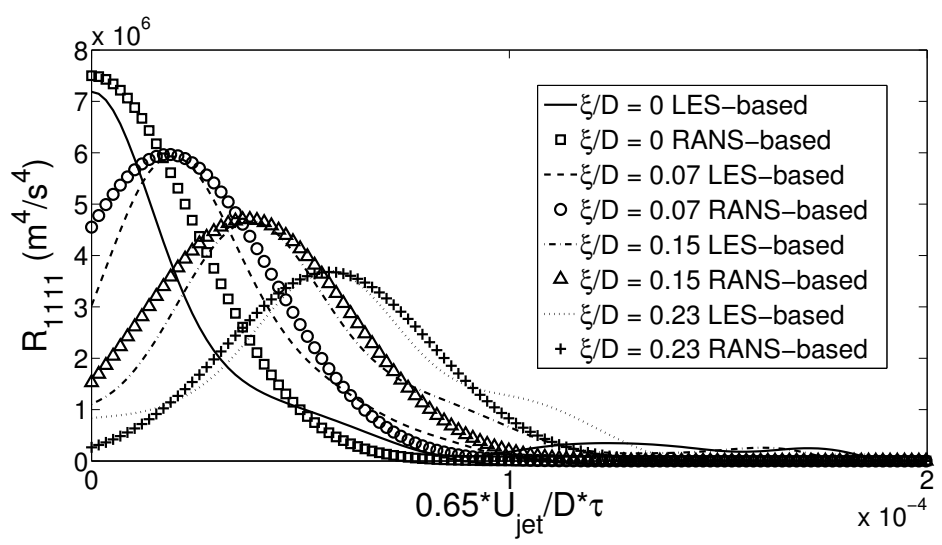

Figure 26: SMC000: $\mathrm{R}_{1111}$ cross-correlation ( $\xi_{1} / \mathrm{D}$ varies, $\xi_{2} / \mathrm{D}=0$ and $\left.\xi_{3} / \mathrm{D}=0\right)$ at $\mathrm{x} / \mathrm{D}$ $=4 ; \mathrm{r} / \mathrm{D}=0.5$ and $\theta=0^{0}$ 


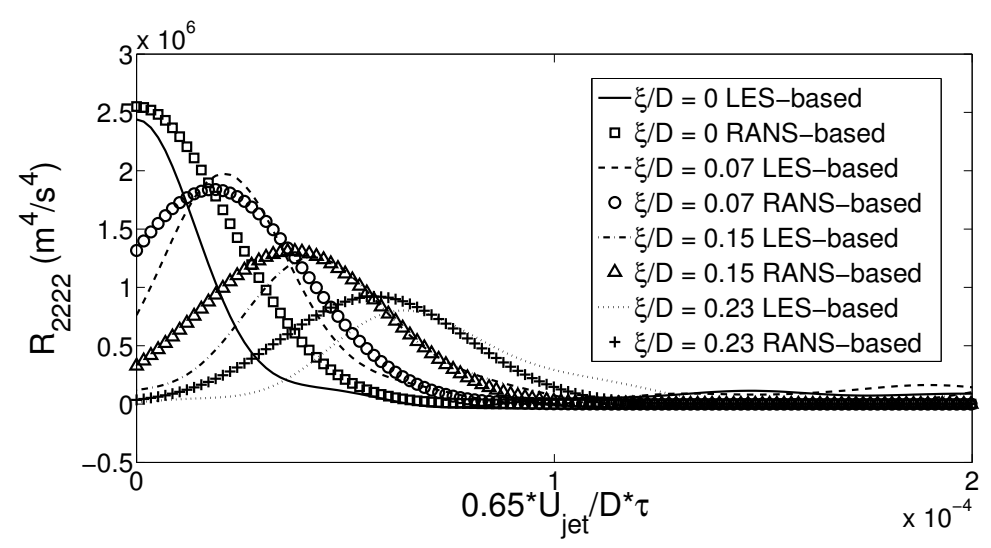

Figure 27: SMC006: $\mathrm{R}_{2222}$ cross-correlation $\left(\xi_{1} / \mathrm{D}=0, \xi_{2} / \mathrm{D}\right.$ varies and $\left.\xi_{3} / \mathrm{D}=0\right)$ at $\mathrm{x} / \mathrm{D}$ $=4 ; \mathrm{r} / \mathrm{D}=0.5$ and $\theta=0^{0}$

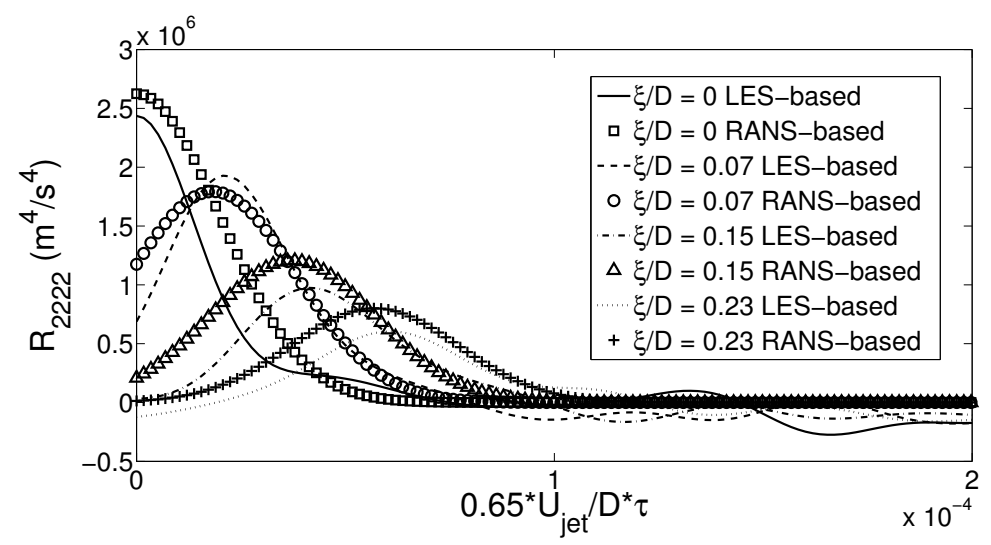

Figure 28: SMC000: $\mathrm{R}_{2222}$ cross-correlation $\left(\xi_{1} / \mathrm{D}=0, \xi_{2} / \mathrm{D}\right.$ varies and $\left.\xi_{3} / \mathrm{D}=0\right)$ at $\mathrm{x} / \mathrm{D}$ $=4 ; \mathrm{r} / \mathrm{D}=0.5$ and $\theta=0^{0}$

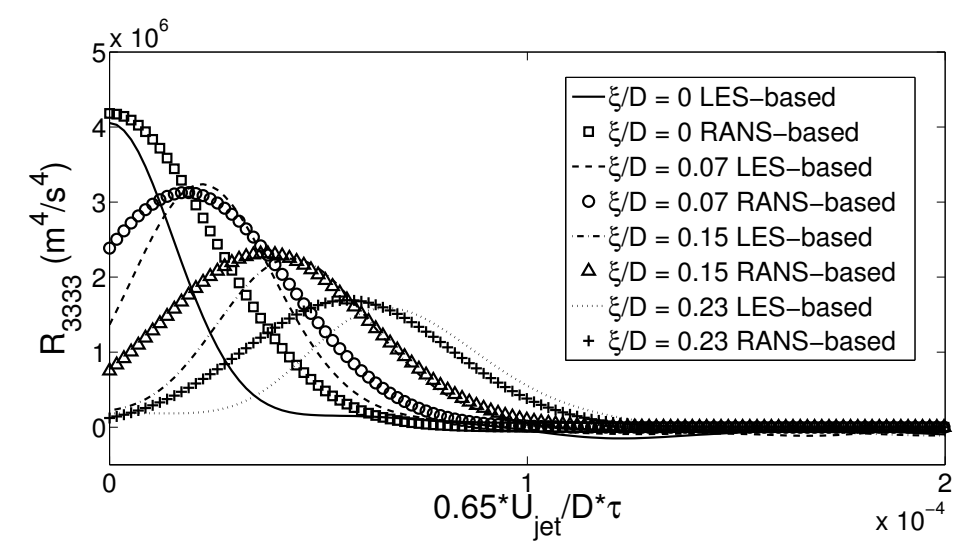

Figure 29: SMC006: $\mathrm{R}_{3333}$ cross-correlation $\left(\xi_{1} / \mathrm{D}=0, \xi_{2} / \mathrm{D}=0\right.$ and $\xi_{3} / \mathrm{D}$ varies $)$ at $\mathrm{x} / \mathrm{D}$ $=4 ; \mathrm{r} / \mathrm{D}=0.5$ and $\theta=0^{0}$ 


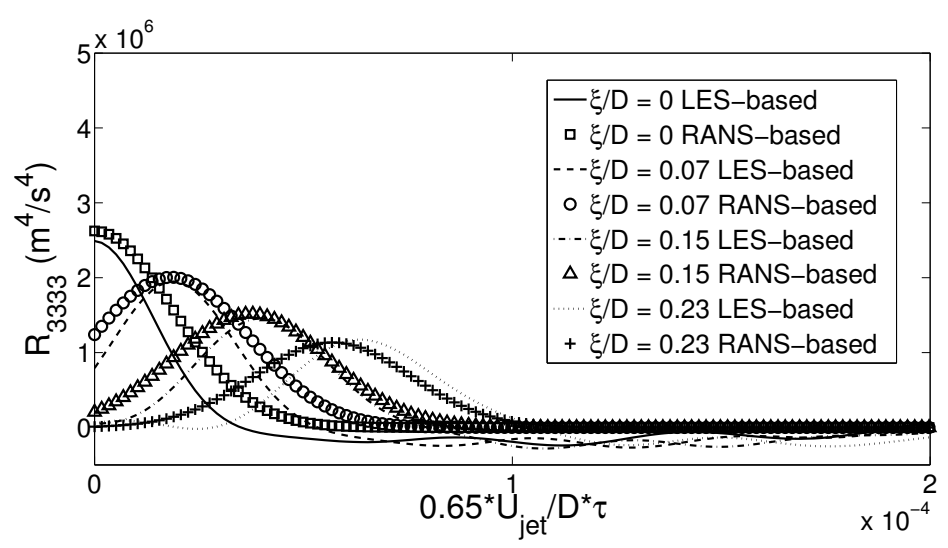

Figure 30: SMC000: $\mathrm{R}_{3333}$ cross-correlation $\left(\xi_{1} / \mathrm{D}=0, \xi_{2} / \mathrm{D}=0\right.$ and $\xi_{3} / \mathrm{D}$ varies $)$ at $\mathrm{x} / \mathrm{D}$ $=4 ; \mathrm{r} / \mathrm{D}=0.5$ and $\theta=0^{0}$

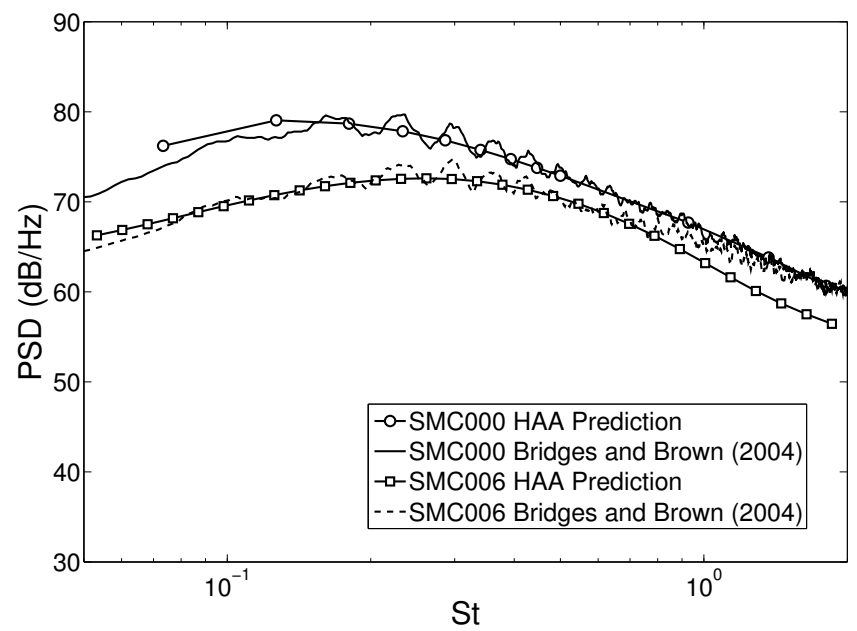

Figure 31: The sound power spectral density (PSD) predictions at $30^{\circ}$ to the jet axis

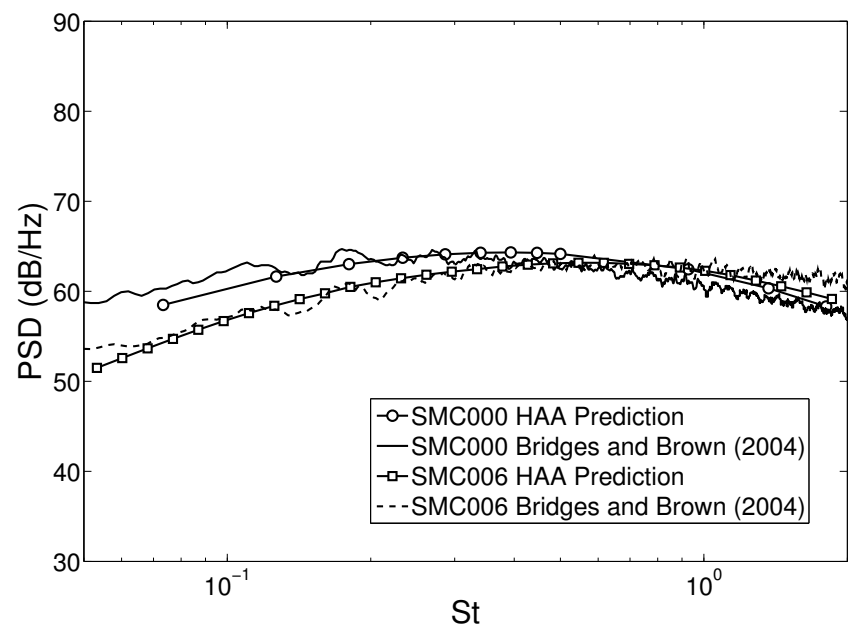

Figure 32: The sound power spectral density (PSD) predictions at $90^{\circ}$ to the jet axis 


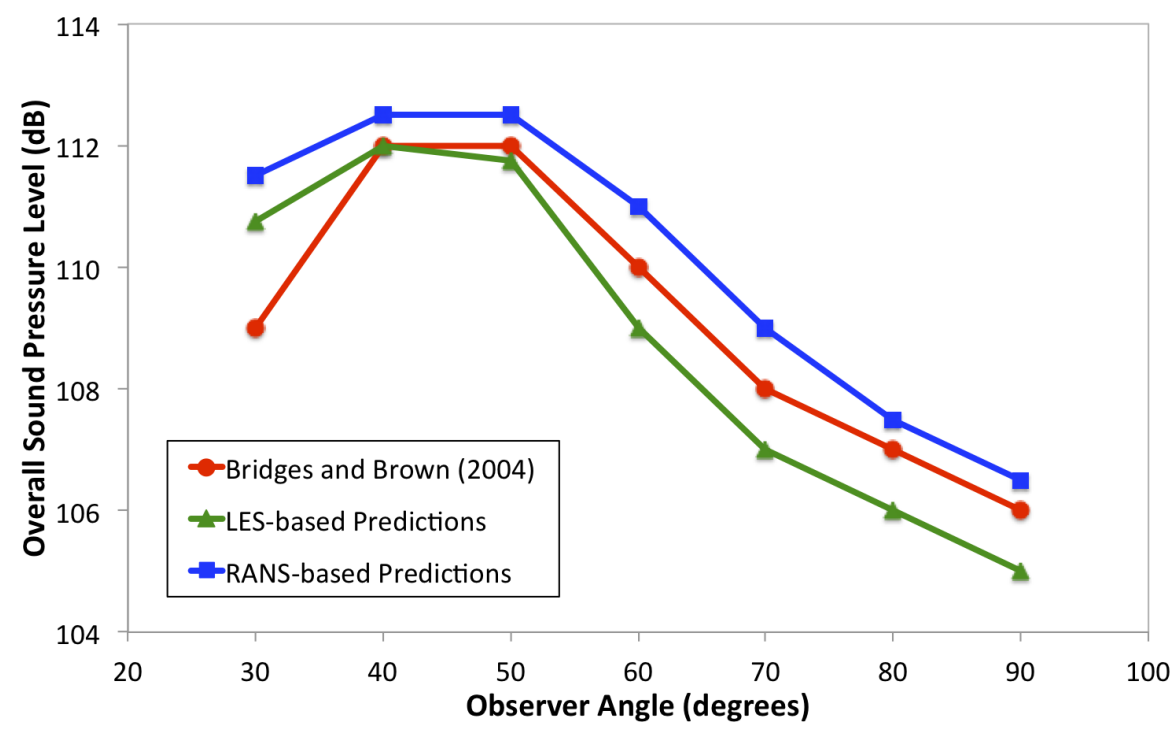

Figure 33: The overall sound pressure level (OASPL) directivity; blue line is the low-order model's (RANS-based) prediction; green line is the high-order model's (LES-based) and red line is the Bridges and Brown ${ }^{1}$ measurements 


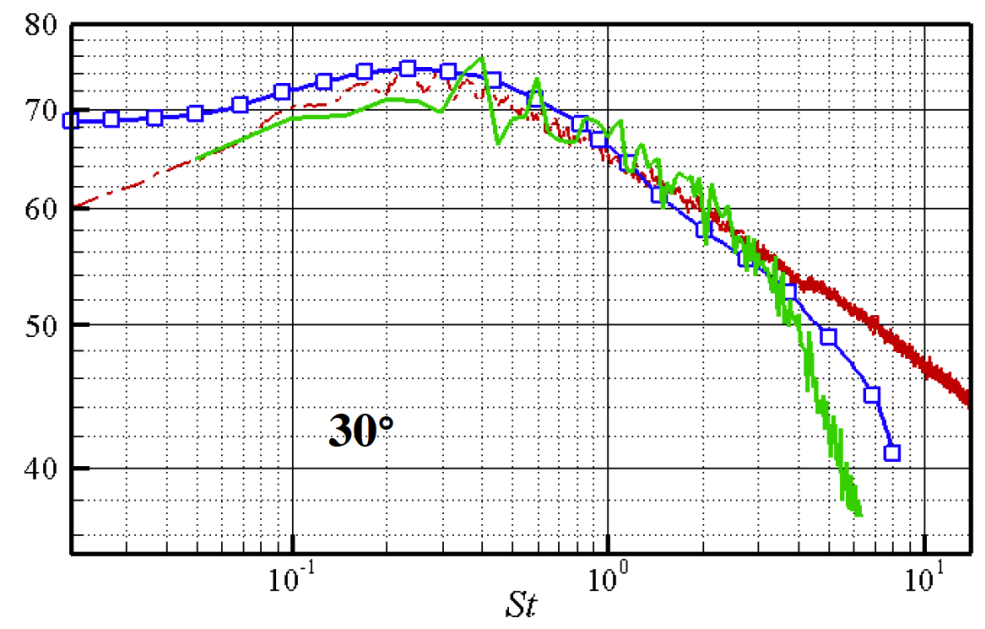

(a) $30^{0}$ to the jet axis

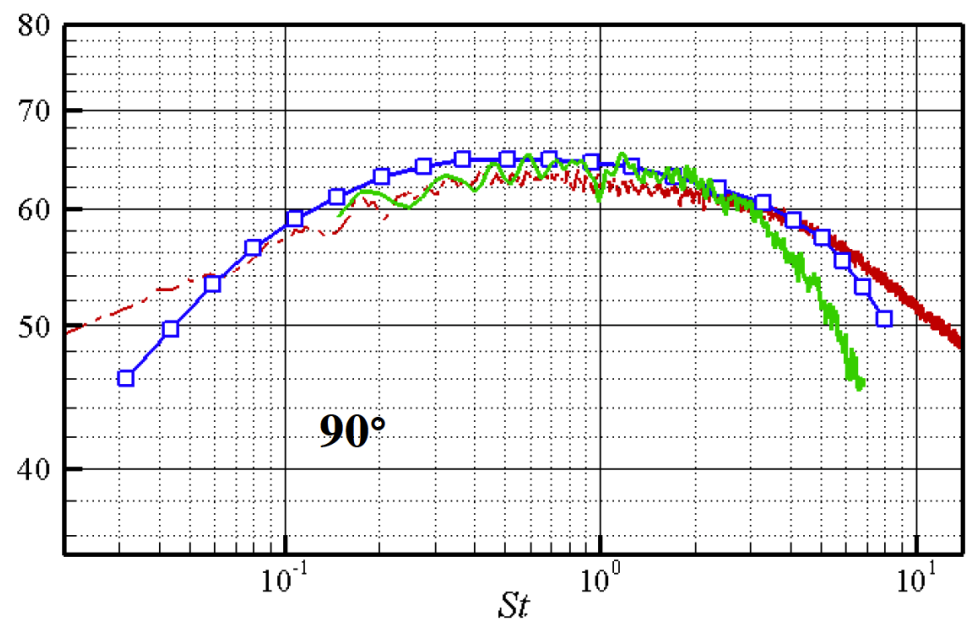

(b) $90^{\circ}$ to the jet axis

Figure 34: Comparison of PSD (dB/St) predictions by the Ffowcs Williams-Hawkings acoustic analogy (green line) (Xia et al. $^{19}$ ) and the low-order model (blue line) with measurements (red line) (Bridges and Brown ${ }^{1}$ ) 


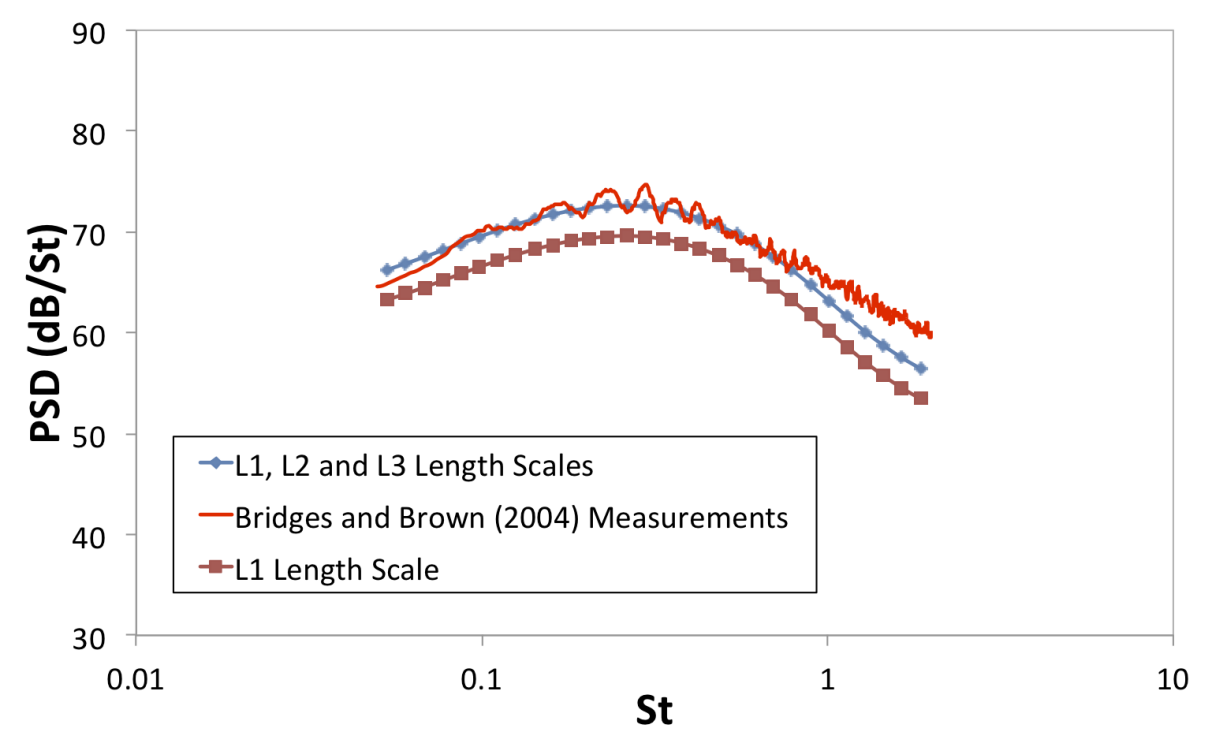

(a) $30^{0}$ to the jet axis

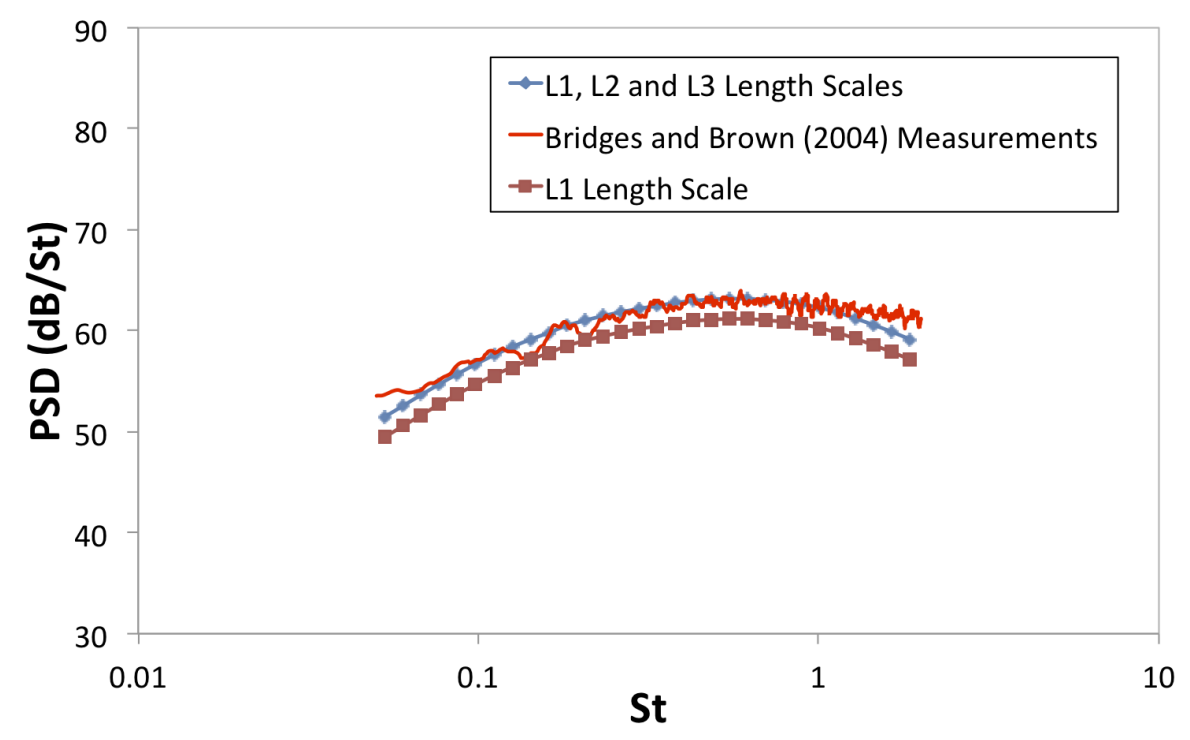

(b) $90^{\circ}$ to the jet axis

Figure 35: Effect of anisotropy of correlation length scales on far-field noise predictions 


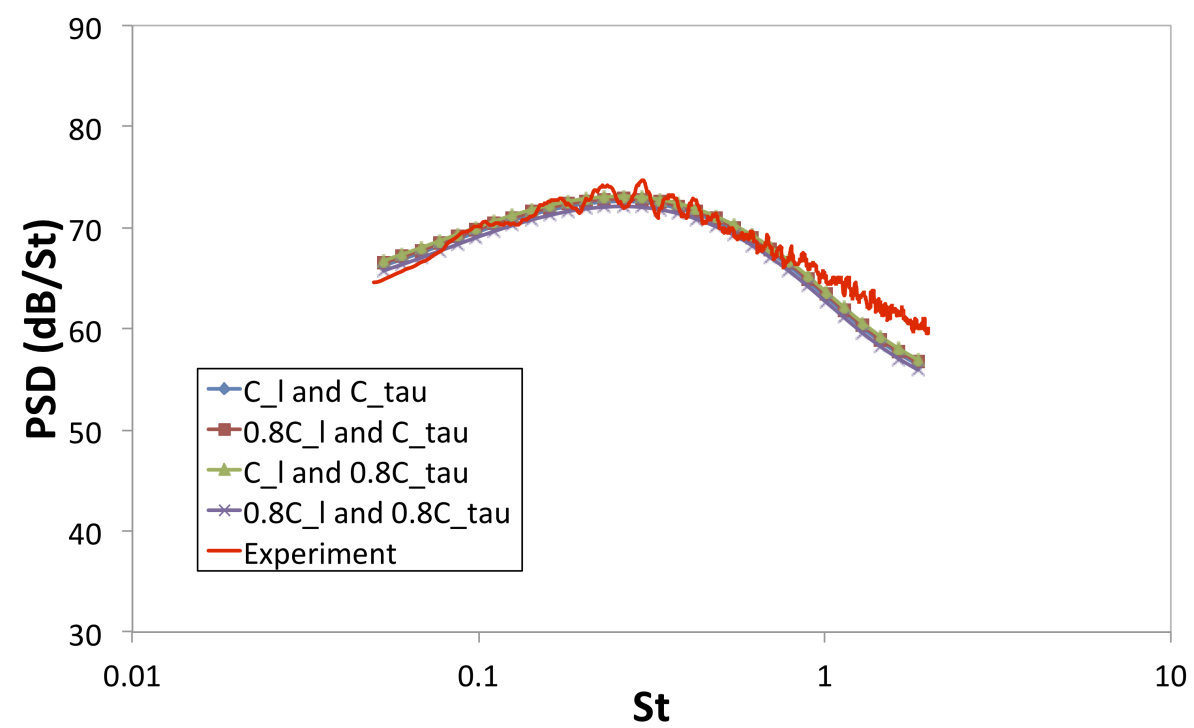

(a) $30^{0}$ to the jet axis

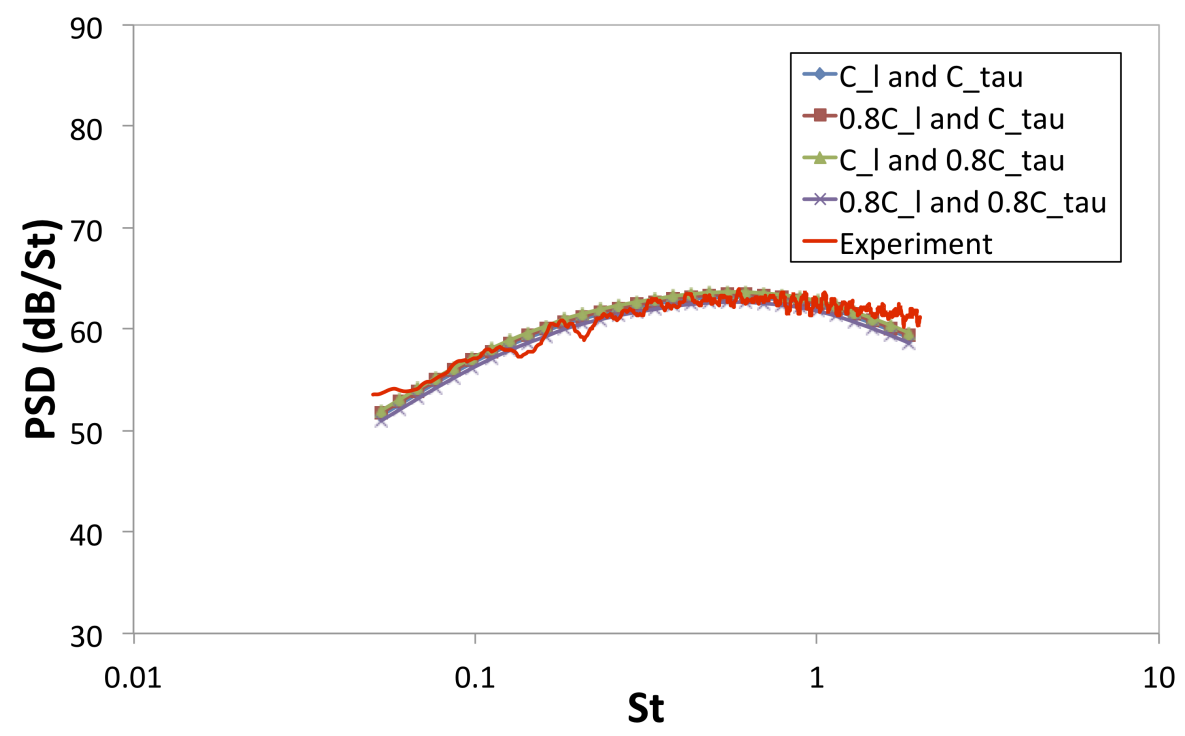

(b) $90^{0}$ to the jet axis

Figure 36: Effect of proportionality constants on far-field noise predictions 


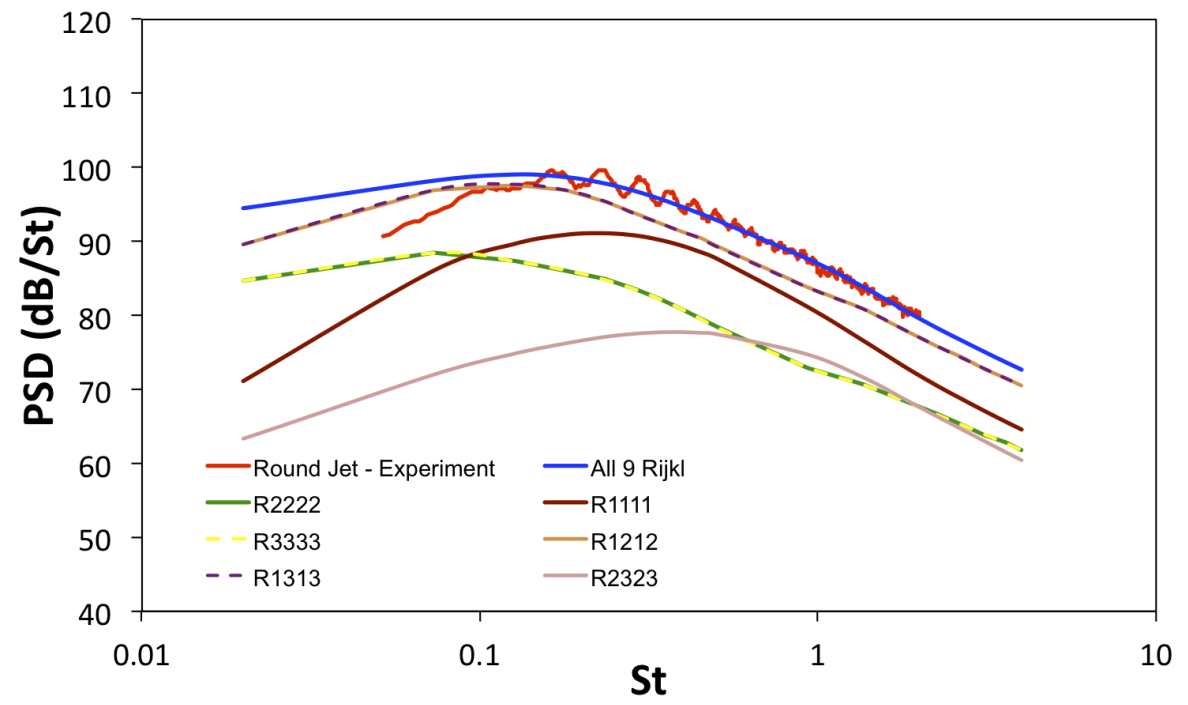

(a) $30^{0}$ to the jet axis

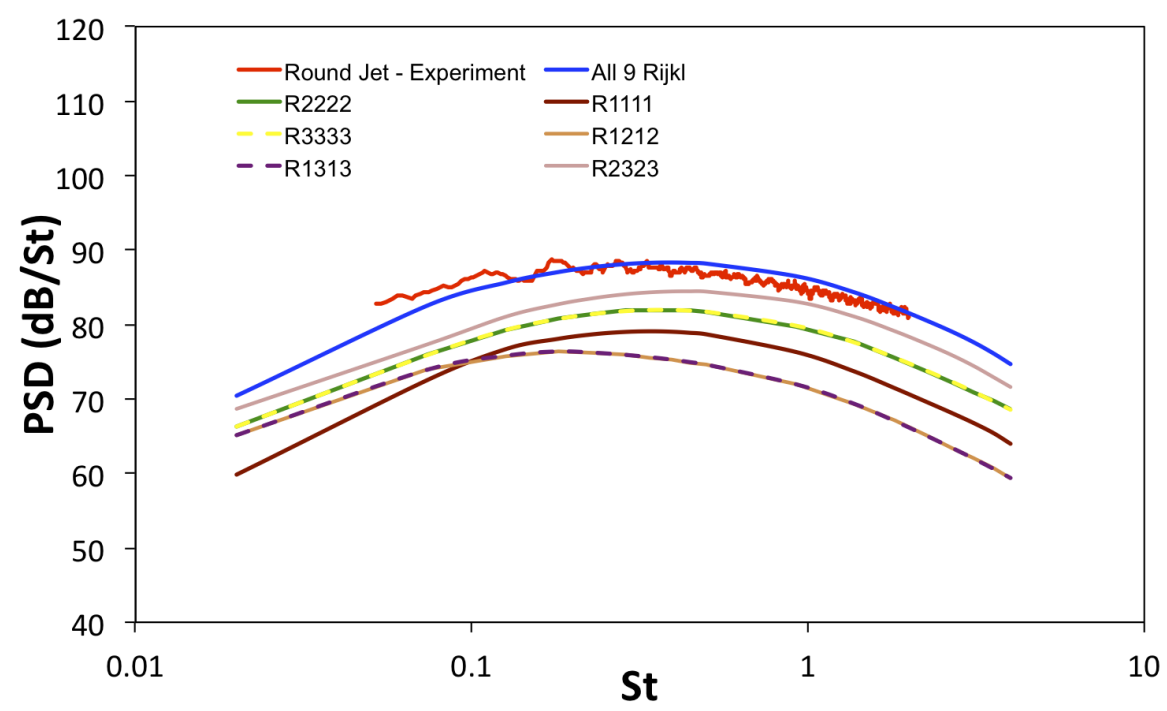

(b) $90^{0}$ to the jet axis

Figure 37: Contribution of the major cross-correlations to the jet noise 


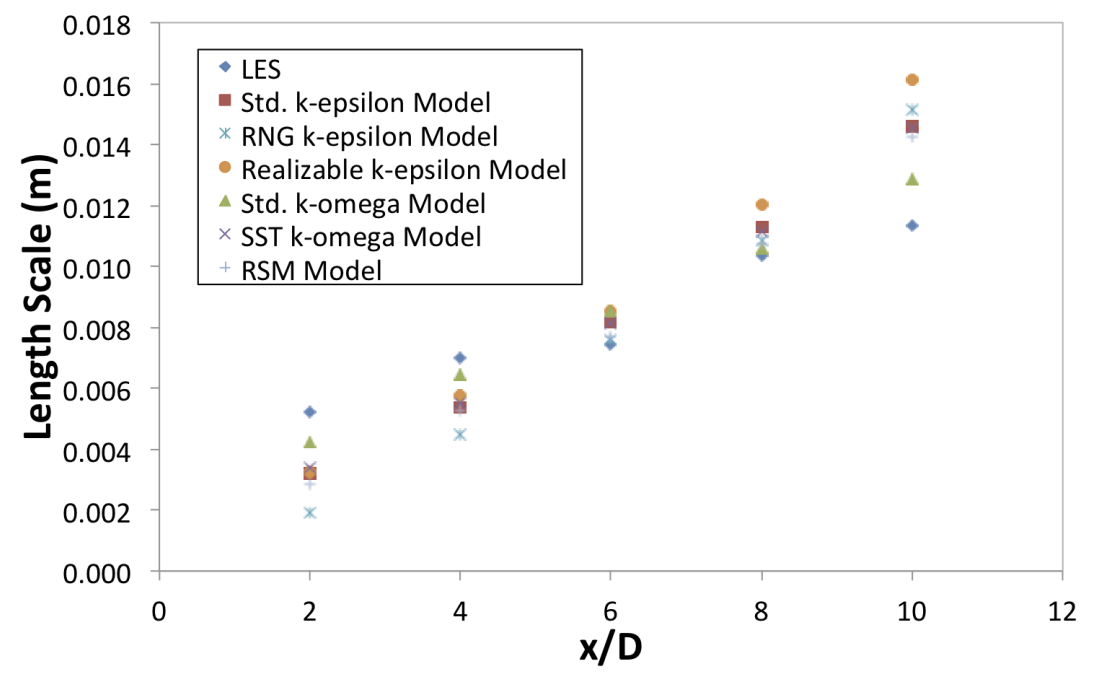

(a) Length Scale

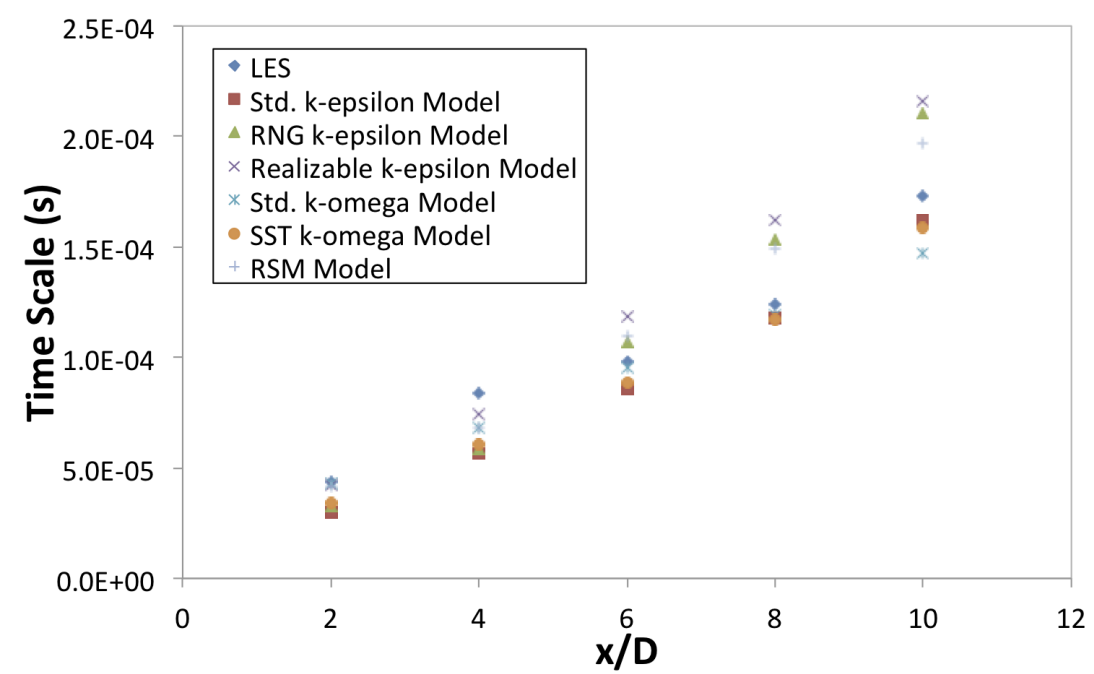

(b) Time Scale

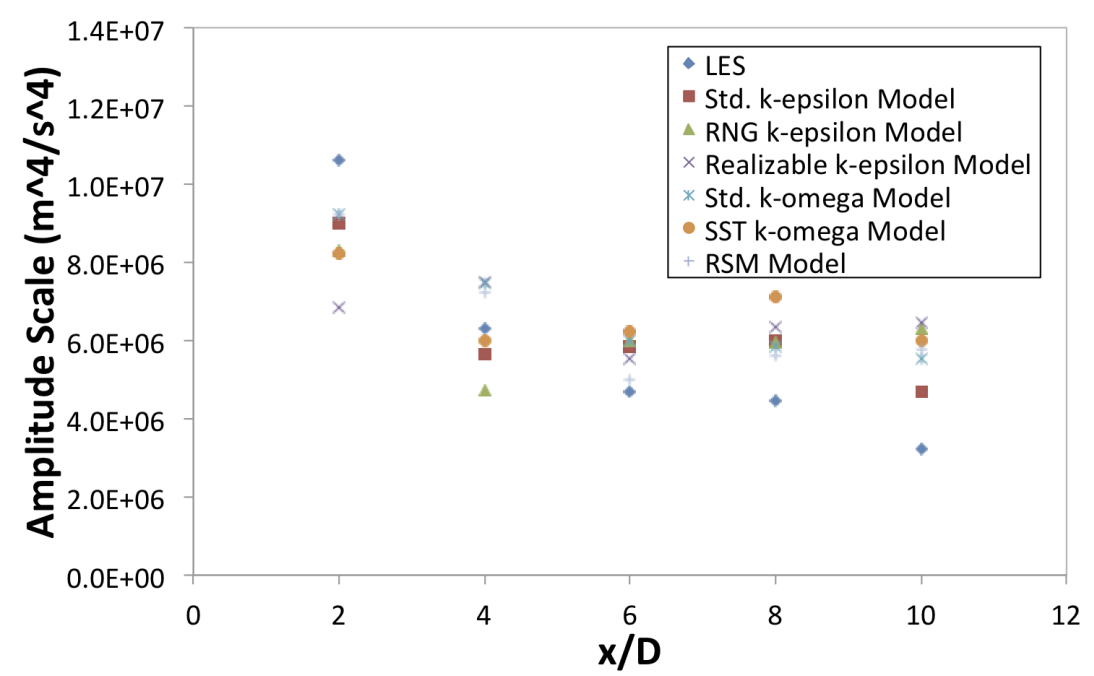

(c) Amplitude Scale

Figure 38: Effect of turbulence models on the correlation length, time and amplitude scales at the jet shear layer $(\mathrm{r}=0.5 \mathrm{D})$ 


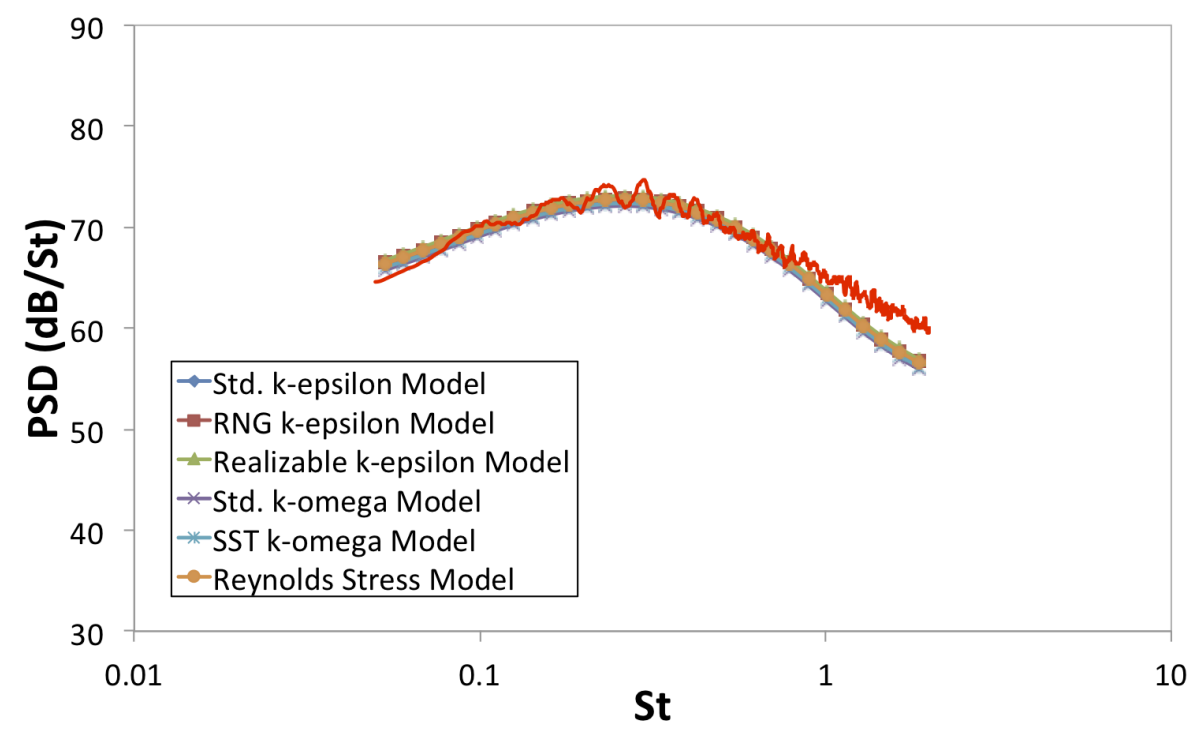

(a) $30^{0}$ to the jet axis

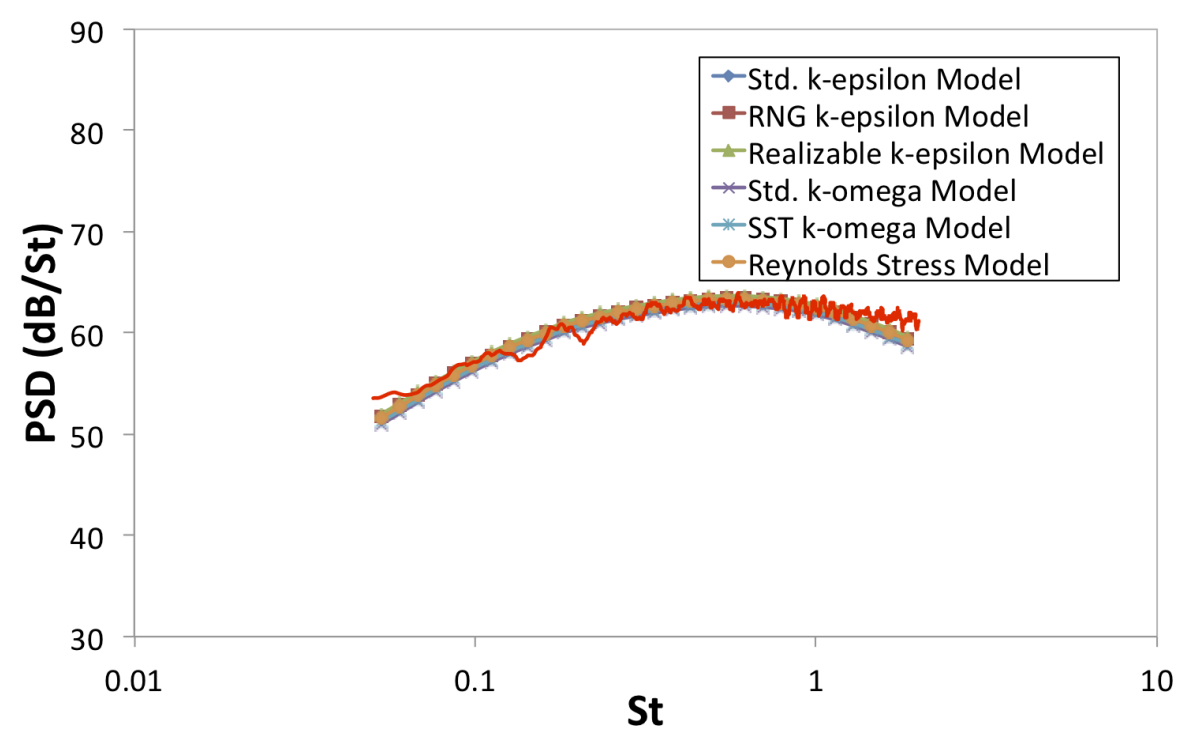

(b) $90^{0}$ to the jet axis

Figure 39: Effect of turbulence models on far-field noise predictions 